\title{
An Alliance of Gel-Based and Gel-Free Proteomic Techniques Displays Substantial Insight Into the Proteome of a Virulent and an Attenuated Histomonas meleagridis Strain
}

\author{
Andreas Monoyios ${ }^{1}$, Karin Hummel ${ }^{2}$, Katharina Nöbauer ${ }^{2}$, Martina Patzl ${ }^{3}$, \\ Sarah Schlosser ${ }^{2}$, Michael Hess ${ }^{1,4}$ and Ivana Bilic ${ }^{1 *}$ \\ ${ }^{1}$ Clinic for Poultry and Fish Medicine, Department for Farm Animals and Veterinary Public Health, University of Veterinary \\ Medicine Vienna, Vienna, Austria, ${ }^{2}$ VetCore Facility for Research, University of Veterinary Medicine Vienna, Vienna, Austria, \\ ${ }^{3}$ Department for Pathobiology, Institute of Immunology, University of Veterinary Medicine Vienna, Vienna, Austria, ${ }^{4}$ Christian \\ Doppler Laboratory for Innovative Poultry Vaccines, University of Veterinary Medicine Vienna, Vienna, Austria
}

OPEN ACCESS

Edited by:

Tiago W. P. Mineo,

Federal University of Uberlandia, Brazi

Reviewed by:

Igor Cestari,

McGill University, Canada

Helioswilton Sales-Campos, Universidade Federal de Goiás, Brazil

${ }^{*}$ Correspondence:

Ivana Bilic

ivana.bilic@vetmeduni.ac.at

Specialty section:

This article was submitted to

Parasite and Host,

a section of the journal

Frontiers in Cellular and Infection

Microbiology

Received: 27 July 2018

Accepted: 30 October 2018

Published: 16 November 2018

Citation:

Monoyios A, Hummel K, Nöbauer K,

Patzl M, Schlosser S, Hess M and Bilic I (2018) An Alliance of Gel-Based and Gel-Free Proteomic Techniques

Displays Substantial Insight Into the

Proteome of a Virulent and an Attenuated Histomonas meleagridis
The unicellular protozoan Histomonas meleagridis is notorious for being the causative agent of histomonosis, which can cause high mortality in turkeys and substantial production losses in chickens. The complete absence of commercially available curative strategies against the disease renders the devising of novel approaches a necessity. A fundamental step toward this objective is to understand the flagellate's virulence and attenuation mechanisms. For this purpose we have previously conducted a comparative proteomic analysis of an in vitro cultivated virulent and attenuated histomonad parasite using two-dimensional electrophoresis and MALDI-TOF/TOF. The current work aimed to substantially extend the knowledge of the flagellate's proteome by applying 2D-DIGE and sequential window acquisition of all theoretical mass spectra (SWATH) MS as tools on the two well-defined strains. In the gel-based experiments, 49 identified protein spots were found to be differentially expressed, of which 37 belonged to the in vitro cultivated virulent strain and 12 to the attenuated one. The most frequently identified proteins in the virulent strain take part in cytoskeleton formation, carbohydrate metabolism and adaptation to stress. However, post-translationally modified or truncated ubiquitous cellular proteins such as actin and GAPDH were identified as upregulated in multiple gel positions. This indicated their contribution to processes not related to cytoskeleton and carbohydrate metabolism, such as fibronectin or plasminogen binding. Proteins involved in cell division and cytoskeleton organization were frequently observed in the attenuated strain. The findings of the gel-based studies were supplemented by the gel-free SWATH MS analysis, which identified and quantified 42 significantly differentially regulated proteins. In this case proteins with peptidase activity, metabolic proteins and actin-regulating proteins were the most frequent findings in the virulent strain, while proteins involved in hydrogenosomal carbohydrate metabolism dominated the results in the attenuated one.

Keywords: Histomonas meleagridis, protozoa, 2D-DIGE, SWATH MS, attenuation, virulence factors, comparative proteomic analysis 


\section{INTRODUCTION}

The unicellular microaerophilic poultry parasite Histomonas meleagridis, first classified in 1920 (Tyzzer, 1919), is a member of the order Tritrichomonadida (Cepicka et al., 2010). In gallinaceous birds, the flagellate is responsible for histomonosis, also known as infectious enterohepatitis or blackhead disease (reviewed in Hess, 2017). In the course of the disease the parasite invades the caecum, then it gains access to the circulatory system through which it travels to the liver and other organs. The necrosis of the liver, which is frequently seen in turkeys, can lead to high mortality (reviewed in McDougald, 2005). In chickens, this gross pathological finding is less common, but severe clinical signs including significant drop in egg production were recorded (Hess and McDougald, 2013). In recent years histomonosis re-emerged, due to the withdrawal of previously effective anti-histomonad drugs and the gaining popularity of free-range poultry farming (reviewed in Hess et al., 2015).

The establishment of a mono-eukaryotic $H$. meleagridis culture derived from a single cell and its successful stable attenuation after numerous in vitro passages opened the road for the development of innovative strategies against the flagellate (Hess et al., 2006; Sulejmanovic et al., 2013). Such attenuated parasites which are propagated in vitro in a mixed bacterial content, termed as xenic culture, were proven to be safe and effective as a vaccine (Liebhart et al., 2011, 2013). Among the known prevention and therapeutic approaches that have been considered against $H$. meleagridis, live attenuated vaccination was recognized to be the most promising, albeit not yet commercially available (reviewed in Liebhart et al., 2017). The further development of the mono-eukaryotic culture by replacing the mixed bacterial flora with Escherichia coli DH5 $\alpha$, showed no effect on the attenuation process (Ganas et al., 2012) and was an important tool for detailed molecular studies.

Despite its importance for poultry health only limited knowledge is available on the parasite's molecular biology, a precondition to develop new protection strategies or improve existing ones. A number of molecular studies determined the phylogenetic relationship of $H$. meleagridis and enriched the collection of available sequences (reviewed in Hess et al., 2015). A non-comparative two-dimensional electrophoresis (2-DE) study on $H$. meleagridis proteome revealed 17 out of 19 identified proteins as actin (Pham et al., 2016). A recent comparative 2-DE proteome analysis of the virulent and attenuated $H$. meleagridis

\footnotetext{
Abbreviations: 2-DE, two-dimensional electrophoresis; 2D-DIGE, twodimensional differential gel electrophoresis; DTT, dithiothreitol; ECM, extracellular matrix; ESI, electrospray ionization; FBAL, fructose-bisphosphate aldolase; Fd, ferredoxin; FDR, false discovery rate; FN, fibronectin; GAPDH, glyceraldehyde-3-phosphate dehydrogenase; HPLC, high performance liquid chromatography; IAA, iodoacetamide; IEF, isoelectric focusing; IPG, immobilized $\mathrm{pH}$ gradient; IS, internal standard; LC-MS/MS, liquid chromatography coupled to tandem mass spectrometry; MALDI-TOF/TOF MS, matrix-assisted laser desorption/ionization time-of-flight/time-of-flight mass spectrometry; $M_{\mathrm{r}}$, molecular mass; NK, Natural killer cells; PEPCK, phosphoenolpyruvate carboxykinase; PFOR, pyruvate ferredoxin oxidoreductase; PFP, pore-forming protein; PLG, plasminogen; SDS-PAGE, sodium dodecyl sulfate polyacrylamide gel electrophoresis; SOD, superoxide dismutase; SPB, surfactant protein B; SWATH, sequential window acquisition of all theoretical mass spectra.
}

culture, identified 49 significantly differentially expressed protein spots between the two strains, corresponding to 31 unique H. meleagridis proteins (Monoyios et al., 2018). In that study, mass spectrometric identification of significant protein spots was supported by the recent de novo transcriptome sequencing of the virulent and attenuated parasite, which was the latest contribution toward the enrichment of $H$. meleagridis sequence data (Mazumdar et al., 2017).

As the evolution in mass spectrometric identification methods and gel image analysis continued, the popularity of 2-DE has risen and the technique was favored for the separation of complete proteins at high resolution (reviewed in Rabilloud et al., 2010). The introduction of immobilized $\mathrm{pH}$ gradient (IPG) gel strips and a new 2-DE protocol, offered the advantage of reproducibility (Görg et al., 2009). However, with conventional 2-DE, only one protein sample can be visualized per gel, while reproducibility issues remained due to the gel-to-gel variation (reviewed in Miller, 2012). From this aspect, the two-dimensional differential gel electrophoresis (2D-DIGE) was considered to be an improvement of 2-DE since it allows for the resolution of three different samples in parallel on the same gel, which are labeled with different fluorescent dyes (reviewed in Miller, 2012). These can include two protein samples to be compared and an internal standard (IS). The latter is used to quantitatively normalize all protein spots, which are included in the experiment, a feature that increases accuracy in detecting differential protein expression (reviewed in Baggerman et al., 2005). In addition, the sensitivity of the 2-DE silver staining protocol and 2DDIGE minimal labeling are unparalleled since the former can detect $1 \mathrm{ng}$ of protein per $2 \mathrm{D}$ spot and the latter as low as $0.1 \mathrm{ng}$ of protein per $2 \mathrm{D}$ spot (reviewed in Sitek et al., 2006).

Nevertheless, from all the proteins present in a complex sample, only $30-50 \%$ can be resolved in a $2-\mathrm{DE}$ gel depending on their abundance and physicochemical properties (reviewed in Baggerman et al., 2005). As a consequence the same group of proteins will be repeatedly visualized and a "déjà vu" of reported results, can be expected (Petrak et al., 2008). Some of the limitations of gel-based methods can be tackled by shotgun proteomic techniques that have the capability of identifying over 10,000 proteins in a sample through a data-dependent approach (Huang et al., 2015). Nonetheless, even with the current technology, challenges arise when reproducible, sensitive, and accurate quantification is required among multiple samples that cover wider proportions of the proteome (Gillet et al., 2012). Acquisition of MS/MS data by the Triple-TOF high-resolution mass spectrometer allowed the development of a new quantification approach called sequential window acquisition of all theoretical mass spectra (SWATH), which is a data-independent acquisition method with the aim of analyzing the fragmentation products of all ions (reviewed in Crutchfield et al., 2016). Label-free quantification of protein abundance with SWATH technology demonstrated high precision, reproducibility, and accuracy (Huang et al., 2015). The usefulness of this approach was recently demonstrated in the comparative proteomic analysis of a fish bacterial pathogen, with the prospect of comprehending its virulence mechanisms 
(Kumar et al., 2016). However, to the extent of our knowledge very few, if any, comparative proteomic studies on protozoa have utilized the SWATH MS technology.

In the present study, 2D-DIGE gel-based experiments were complemented by the gel-free, label-free proteomic technique which involved nano-scale liquid chromatography coupled with a hybrid high-resolution mass spectrometer and SWATH MS technology. The main objective was to resolve proteome differences or similarities between the in vitro cultivated virulent and attenuated $H$. meleagridis strains in greater detail in a comprehensive setting.

\section{MATERIALS AND METHODS}

\section{Cultivation and Collection of Virulent and Attenuated $\boldsymbol{H}$. meleagridis Parasites}

The experiments were performed using virulent and attenuated $H$. meleagridis parasites which were propagated in vitro as monoxenic mono-eukaryotic cultures designated as $H$. meleagridis/turkey/Austria/2922-C6/04-10x/DH5 $\alpha$ and $H$. meleagridis/turkey/Austria/2922-C6/04-290x/DH5 $\alpha$, respectively (Ganas et al., 2012). The incubation took place at $41^{\circ} \mathrm{C}$ in a standard medium consisting of Medium 199 (Gibco ${ }^{\mathrm{TM}}$, Vienna, Austria), 15\% heat-inactivated fetal bovine serum $\left(\mathrm{Gibco}^{\mathrm{TM}}\right)$, and $0.22 \%$ of sterilized rice starch (Carl Roth $\mathrm{GmbH}+$ Co. KG, Karlsruhe, Germany). Parasites were passaged every 3 days (Ganas et al., 2012) and at the final passage $1 \mathrm{ml}$ of $E$. coli DH5 $\alpha$ liquid culture (colony forming units (CFU): $5 \times$ $10^{8}$ bacterial cells $/ \mathrm{ml}$ ), which was grown overnight at $37^{\circ} \mathrm{C}$, was added to the cultures. Subsequently, the virulent lowpassaged parasites were harvested at passage number 25 and the attenuated high-passaged parasites at passage number 303 . A purification protocol consisting of consecutive washing and centrifugation steps was used to collect $H$. meleagridis cells and to remove most of the bacteria as previously described (Monoyios et al., 2018). Pellets of collected parasites were stored at $-80^{\circ} \mathrm{C}$ until further use. The quality of purification and parasite numbers were evaluated using Trypan Blue Stain (Gibco $^{\mathrm{TM}}$, Invitrogen, Vienna, Austria) as previously described (Zaragatzki et al., 2010).

\section{Protein Sample Preparation for 2D-DIGE and SWATH MS}

In total four biological replicates and four technical replicates were used in the 2D-DIGE experiment. For each sample protein was extracted from $6 \times 10^{7}$ purified parasites, which were cocultivated in tissue culture flasks with E. coli DH5 $\alpha$. Four separate virulent and attenuated $H$. meleagridis cultures were harvested on the same day at passage number 25 and 303, respectively (Figure S1). Two parasite cultures from each strain were pooled together prior to the protein extraction resulting in two samples for the virulent strain $25 \mathrm{a}$ and $25 \mathrm{~b}$ and two for the attenuated strain 303a and 303b (Figure S1). In that way, a higher number of parasites was obtained and at the same time higher biological variability was introduced in each sample. In order to obtain a second group of four protein samples (25a, 25b, 303a, 303b), the cultivation and the extraction schemes from above were repeated using $H$. meleagridis parasites, which were cultivated and harvested at a different time point. For both experiments, protein extraction of the first biological replicate was performed on the day 1 (sample a) and the second biological replicate on the day 2 (sample b), and as a result four protein samples were included in each of the two 2D-DIGE experiments: 25a, 25b, 303a, 303b (Figure S1). In each 2D-DIGE experiment, each of the two biological replicates of a given strain (e.g., 25a and 25b) was swap labeled with the fluorescent dyes (e.g., 25a with G-Dye200 and 25a with G-Dye300, Table 1) and the acquired fluorescent images represented technical replicates for the 2DDIGE protocol (Figure S1). The protein sample preparation procedure, described below, was applied to all eight samples. Two pellets of collected parasites were resuspended in extraction buffer [50 mM Tris-HCl pH 8.8, 5 mM EDTA, $100 \mathrm{mM} \mathrm{KCl}$, $1 \%(\mathrm{w} / \mathrm{v})$ dithiothreitol (DTT)] containing a complete protease inhibitor cocktail (Roche Applied Science, Roche Diagnostics, Mannheim, Germany) and sonicated (power: 30\%, duration: 10 s, cycle (pulsation): $5 \times 10 \%$, Bandelin Sonopuls HD2070, Bandelin electronic, Berlin, Germany) three times on ice with a $30 \mathrm{~s}$ rest period in between. Consequently, a centrifugation step was performed at $18,000 \times \mathrm{g}$ for $15 \mathrm{~min}$ at $4^{\circ} \mathrm{C}$. The pellets containing rice starch particles were discarded. The supernatants were then mixed with lysis buffer ( $\mathrm{pH}: 8.5)$ containing $7 \mathrm{M}$ urea, $2 \mathrm{M}$ thiourea, $4 \%$ (w/v) CHAPS, $30 \mathrm{mM}$ Tris- $\mathrm{HCl}$ (Arnal et al., 2015) to which complete protease inhibitor cocktail was added. Protein concentration was measured using the 2-D Quant kit (GE Healthcare Life Sciences, Sigma-Aldrich Handels $\mathrm{GmbH}$, Vienna, Austria) based on the manufacturer's instructions. The 2-D Clean up kit (GE Healthcare Life Sciences) was used for further protein purification. The resulting protein pellet was resuspended in the lysis buffer described above and stored at $-80^{\circ} \mathrm{C}$.

The first group of four protein samples was used for the shotgun proteomic analysis and in a 2D-DIGE experiment, designated as $2 \mathrm{D}$-DIGE- $\alpha$. The second group was used in a repetition of the $2 \mathrm{D}$-DIGE experiment, referred to as 2D-DIGE- $\beta$.

\section{Protein Labeling With G-Dye Fluorophores and 2D-DIGE Protocol}

The protein concentration of all four protein samples was measured with the 2-D Quant kit to ensure accurate sample application and labeling. The G-Dye100 (G100), G-Dye200 (G200), and G-Dye300 (G300) lysine fluorescent dyes, included in the Refraction-2D ${ }^{\mathrm{TM}}$ kit (NH DyeGNOSTICS GmbH, Halle, Germany) were reconstituted and used for the minimal labeling of protein samples according to manufacturer's instructions. Each 2D-DIGE experiment consisted of four 2-DE gels. In each gel, three samples were visualized, namely $25 \mathrm{a}$ or $25 \mathrm{~b}, 303 \mathrm{a}$ or $303 \mathrm{~b}$ and the IS (Table 1). The IS sample, labeled with GDye100, contained an equal amount of all four protein samples. To exclude preferential labeling bias, the four protein samples were individually labeled with 400 pmol of G-Dye200 or GDye300 dye using dye-swap correction (Table 1). In each gel, 150 $\mu \mathrm{g}$ of protein was resolved in the first dimension by conducting isoelectric focusing (IEF) and in the second dimension by SDSPAGE. 
TABLE 1 | Experimental design for the 2D-DIGE- $\alpha$ and 2D-DIGE- $\beta$ experiments.

\begin{tabular}{lccc}
\hline Gel Nr. & \multicolumn{3}{c}{ Fluorescent dyes } \\
\cline { 2 - 4 } & G-Dye100 & G-Dye200 & G-Dye300 \\
\hline 1 & IS $(50 \mu \mathrm{g})$ & $25 \mathrm{a}(50 \mu \mathrm{g})$ & $303 \mathrm{~b}(50 \mu \mathrm{g})$ \\
2 & IS $(50 \mu \mathrm{g})$ & $25 \mathrm{~b}(50 \mu \mathrm{g})$ & $303 \mathrm{a}(50 \mu \mathrm{g})$ \\
3 & IS $(50 \mu \mathrm{g})$ & $303 \mathrm{~b}(50 \mu \mathrm{g})$ & $25 \mathrm{a}(50 \mu \mathrm{g})$ \\
4 & IS $(50 \mu \mathrm{g})$ & $303 \mathrm{a}(50 \mu \mathrm{g})$ & $25 \mathrm{~b}(50 \mu \mathrm{g})$ \\
\hline
\end{tabular}

IS, Internal standard.

25a, Biological replicate 1 of virulent Histomonas meleagridis parasites, of passage number 25 , protein extraction on day 1 .

25b. Biological replicate 2 of virulent $\mathrm{H}$. meleagridis parasites, of passage number 25, protein extraction on day 2.

303a, Biological replicate 1 of attenuated H. meleagridis parasites, of passage number 303 , protein extraction on day 1 .

303b, Biological replicate 2 of attenuated $\mathrm{H}$. meleagridis parasites, of passage number 303 , protein extraction on day 2 .

Previous to IEF, $18 \mathrm{~cm}$ IPG strips with a linear $\mathrm{pH}$ range 47 (ReadyStrip $^{\mathrm{TM}}$ IPG strips, Bio-Rad ${ }^{\circledR}$, Vienna, Austria) were rehydrated for $16-17 \mathrm{~h}$ at room temperature in rehydration buffer [7 M urea, $2 \mathrm{M}$ thiourea, $4 \%$ (w/v) CHAPS, $0.002 \%(\mathrm{w} / \mathrm{v})$ bromophenol blue] to which $0.4 \%(\mathrm{w} / \mathrm{v})$ DTT and $0.5 \%(\mathrm{v} / \mathrm{v})$ SERVALYT ${ }^{\mathrm{TM}}$ 3-10 Carrier Ampholytes (Serva Electrophoresis, Heidelberg, Germany) were added (Arnal et al., 2015). The sample application was performed according to the anodic cuploading technique. In the first dimension, the proteins were focused using PROTEAN ${ }^{\circledR}$ IEF Cell (Bio-Rad $\left.{ }^{\circledR}\right)$ at $20^{\circ} \mathrm{C}$ with an electric current limitation of $50 \mu \mathrm{A} / \mathrm{IPG}$ strip. Isoelectric focusing was performed in six steps with the following voltage settings and duration: (i) $150 \mathrm{~V}$ for $1.5 \mathrm{~h}$, (ii) $300 \mathrm{~V}$ for $1.5 \mathrm{~h}$, (iii) $600 \mathrm{~V}$ for $1 \mathrm{~h}$, (iv) linear voltage increase to $8,000 \mathrm{~V}$ for $0.5 \mathrm{~h},(\mathrm{v})$ final focusing step at $8,000 \mathrm{~V}$ for a total of $45,000 \mathrm{Vh}$, (vi) $500 \mathrm{~V}$ resting step. Following this, all four IPG strips that belonged to the same 2D-DIGE experiment were stored at $-80^{\circ} \mathrm{C}$.

Prior to the separation of focused proteins by SDS-PAGE, the reduction of the thawed IPG strips was performed in equilibration buffer (6 M urea, $0.375 \mathrm{M}$ Tris- $\mathrm{HCl} \mathrm{pH} 8.8,2 \%$ SDS, $20 \%$ glycerol) containing $2 \%(\mathrm{w} / \mathrm{v})$ DTT for $15 \mathrm{~min}$ at room temperature. Then the IPG strips were alkylated under the same conditions and in the same equilibration buffer which contained $2.5 \%(\mathrm{w} / \mathrm{v})$ iodoacetamide (IAA) instead of DTT.

For the second dimension, vertical slab gels [resolving gel dimensions: width $\times$ height $\times$ thickness $=18.3 \mathrm{~cm} \times 16.3 \mathrm{~cm}$ $\times 1.5 \mathrm{~mm}, 1.5 \mathrm{M}$ Tris- $\mathrm{HCl} \mathrm{pH} 8.8,10 \%$ SDS, (total monomer concentration) $\mathrm{T}=10 \%$, (percentage of crosslinker) $\mathrm{C}=3.33 \%$ ] were casted simultaneously using the PROTEAN ${ }^{\circledR}$ II multigel casting chamber (Bio-Rad ${ }^{\circledR}$ ) according to manufacturer's instructions. Subsequently, the equilibrated IPG strips were placed on top of a polymerized stacking gel $(18.3 \mathrm{~cm} \times 3 \mathrm{~cm} \times$ $1.5 \mathrm{~mm}$ ) using $0.5 \%$ pre-warmed agarose, containing $0.05 \%$ of bromophenol blue dye, which was used for tracking purposes. The second dimension was carried out using Protean ${ }^{\circledR}$ II xi cell (Bio-Rad ${ }^{\circledR}$ ) with a current of $15 \mathrm{~mA} /$ gel at $4^{\circ} \mathrm{C}$ until the tracking dye ran off the bottom of the gels. Fifteen microliters of molecular marker [Spectra ${ }^{\mathrm{TM}}$ Multicolor Broad Range Protein Ladder, 10$260 \mathrm{kDa}$, Thermo Scientific ${ }^{\mathrm{TM}}$, Fisher Scientific (Austria) GmbH,
Vienna, Austria] was applied for approximate estimation of protein molecular mass $\left(M_{\mathrm{r}}\right)$.

The fluorescent labeled gels were scanned with Typhoon 9400 scanner (GE Healthcare Life Sciences) at a resolution of $100 \mu \mathrm{m}$. After scanning, protein patterns were additionally visualized following published silver staining protocols (Blum et al., 1989; Miller and Gemeiner, 1992).

\section{Computational Image and Statistical Analysis of Fluorescent Proteome Patterns}

Twelve fluorescent gel images were obtained from each 2D-DIGE experiment and included in the computational image (Figure S2) and statistical analysis which was performed with the Delta2D software version 4.7 (Decodon GmbH, Greifswald, Germany) (Berth et al., 2007). The investigations were characterized as multiplex experiments. The 12 fluorescent images were categorized into three groups consisting of four images each. The three groups were termed "virulent 25," "attenuated 303," and "IS." The categorization was performed by assigning gel, sample and channel information to each image. The "in gel standard" warping strategy was chosen to coordinate corresponding spot regions across all gel images and to eliminate positional deviations due to technical reasons. The "union fusion" algorithm was applied to combine all gel images in a single fusion image based on their common region. IS images were excluded from this step as they do not add information to the consensus protein spot pattern of the parasites. Spot detection was performed once on the fusion image. The consensus spot pattern information was transferred to all gel images, including the IS images, allowing for the acquisition of complete expression profiles for a given spot across all 12 fluorescent gel images.

The statistical analysis of expression profiles was carried out with TIGR Multiple Experiment Viewer $(\mathrm{MeV})$ which is included in Delta2D software version 4.7 (Decodon $\mathrm{GmbH}$ ). The IS images were not included in the statistical analysis but were taken into account for the normalization of protein spots visualized on gel images belonging to the virulent (passage number 25) and attenuated (passage number 303) group. In all four gels of a given 2D-DIGE experiment, the same sample served as the IS; therefore, all spots were reliably normalized to the same sample. Hypothesis testing was performed using a permutation Student's $t$-Test by assuming unequal group variances and by applying the following false discovery rate (FDR) correction in order to address the multiple hypothesis testing problem (Benjamini and Hochberg, 1995): with a confidence level of 95\% the number of accepted false positive expression profiles was $\leq 20$. Protein spots with significant $(P<0.05)$ differences in the mean normalized volumes $(\% \mathrm{~V})$, between virulent and attenuated gel image groups, were submitted for identification by MALDI-TOF/TOF or LC-MS/MS.

\section{Identification of Differentially Abundant Protein Spots by MALDI-TOF/TOF}

Significantly $(P<0.05)$ differentially abundant protein spots that could be located on silver-stained fluorescent gels were excised and processed for identification. Protein spots were pooled 
from two or more gels to obtain sufficient material for mass spectrometric analysis. After washing and de-staining, reduction of spots with DTT and alkylation with IAA was conducted (Gharahdaghi et al., 1999; Jiménez et al., 2001). Consequently, in-gel digestion with trypsin (Trypsin Gold, Mass Spectrometry Grade, Promega, Madison, USA) was performed according to Shevchenko et al. (1996). The peptides were then extracted and dried using a vacuum concentrator (Eppendorf, Hamburg, Germany). Afterwards, de-salting and concentrating of tryptic peptides was performed to increase the sensitivity of MS using $\mu$-Zip Tips C18 (Millipore, Billerica, USA) according to the manufacturer's instructions.

A $0.5 \mu \mathrm{l}$ aliquot of de-salted peptides was added to $0.5 \mu \mathrm{l}$ of the matrix solution $[\alpha$-cyano- 4 -hydroxycinnamic acid, saturated in acetonitrile- $0.1 \%$ trifluoroacetic acid (30:70, v/v)] and $1 \mu \mathrm{l}$ of the resulting mixture was spotted on a ground steel MALDI target plate (Bruker Daltonics, Bremen, Germany) and allowed to dry at room temperature. Spectra data were acquired in MS and MS/MS modes by performing MALDI-TOF/TOF MS (Ultraflex II, Bruker Daltonics). FlexAnalysis 3.0 and Biotools 3.2 software (Bruker Daltonics) were used for spectra processing and peak annotation. Processed spectra were submitted to an in-house Mascot server (version 2.3, Matrix Science, Boston, USA) using the ProteinScape 2.1 software (Bruker Daltonics). Subsequent searches were performed in an amino acid database of $E$. coli proteins (UnitProt DB, taxonomy 562 EcolX $^{1}$ ) combined with a database containing conceptually translated contigs from the $H$. meleagridis reference transcriptome (size: 3356 unique contigs; study accession number PRJEB19109²) (Mazumdar et al., 2017) and common Repository of Adventitious Proteins (cRAP) database ${ }^{3}$. The $H$. meleagridis contigs were annotated based on their homology to known genes (Mazumdar et al., 2017). The parameters for the search were: fixed modifications = carbamidomethylation $(\mathrm{C})$; variable modifications = oxidation (M), deamidation (NQ), pyroglutamic acid formation; enzyme specificity $=$ trypsin; charge state $(Z)=+1$; maximum missed cleavages allowed $=2$; peptide mass tolerance $=100$ ppm $(150$ ppm in some cases); fragment mass tolerance $=1.0 \mathrm{Da}$. Mascot identifications having at least one peptide with a Mascot score $>20$ were regarded as statistical significant $(P<0.05)$, while in ProteinScape 2.1 software, protein scores $>80$ were considered as significant.

\section{Identification of Differentially Abundant Protein Spots by LC-MS/MS}

Five and three protein spots from the 2D-DIGE- $\alpha$ and 2DDIGE- $\beta$ experiment, respectively, could not be identified by MALDI-TOF/TOF MS and were analyzed by LC-MS/MS. For this, proteolytically generated peptides were separated by a high-performance chromatography system (nano-HPLC Ultimate 3000 RSLC system, Dionex) and analyzed with a high-resolution hybrid triple quadrupole time-of-flight mass

\footnotetext{
${ }^{1}$ http://www.uniprot.org/

${ }^{2}$ http://www.ebi.ac.uk/ena/data/view/PRJEB19109

${ }^{3} \mathrm{ftp}: / / \mathrm{ftp}$.thegpm.org/fasta/cRAP/crap.fasta
}

spectrometer (TripleTOF 5600+, Sciex, USA) connected via nano-electrospray ionization (ESI) interface.

Remainders of the de-salted peptides after in-gel digest were evaporated and reconstituted in $0.1 \%$ aqueous TFA for injection into LC-MS. Sample pre-concentration and de-salting was accomplished with a $5 \mathrm{~mm}$ Acclaim PepMap $\mu$-Precolumn $(300 \mu \mathrm{m}$ inner diameter, $5 \mu \mathrm{m}$ particle size, and $100 \AA$ pore size) (Dionex). As a mobile phase $2 \%$ ACN in ultra-pure $\mathrm{H}_{2} \mathrm{O}$ with $0.05 \%$ TFA was used for loading and de-salting of peptide samples with a flow rate of $5 \mu \mathrm{l} / \mathrm{min}$. For peptide separation a $25 \mathrm{~cm}$ Acclaim PepMap C18 column $(75 \mu \mathrm{m}$ inner diameter, $3 \mu \mathrm{m}$ particle size, and $100 \AA$ pore size) was used operated at a flow rate of $300 \mathrm{nl} / \mathrm{min}$. The gradient started with $4 \%$ B ( $80 \%$ ACN with $0.1 \%$ formic acid) and increased to $35 \%$ $\mathrm{B}$ in $60 \mathrm{~min}$. It was followed by a washing step with $90 \% \mathrm{~B}$. Mobile Phase A consisted of ultra-pure $\mathrm{H}_{2} \mathrm{O}$ with $0.1 \%$ formic acid. Mass spectra of HPLC-separated peptides were acquired using an information dependent data acquisition mode (IDA). MS1 spectra were collected in the range of $400-1,500 \mathrm{~m} / \mathrm{z}$ for $250 \mathrm{~ms}$. The 25 most intense precursors with charge state 24, which exceeded 100 counts per second, were selected for fragmentation. MS2 spectra were acquired in the range of 100$1,800 \mathrm{~m} / \mathrm{z}$ for $110 \mathrm{~ms}$. Precursor ions were dynamically excluded from reselection for $10 \mathrm{~s}$. The nano-HPLC system was operated by Chromeleon 6.8 using DCMS-Link (Dionex) and the MS by Analyst Software 1.6 (Sciex).

Database searches of raw data were performed using ProteinPilot 5.0 (Sciex) in the combined database of E. coli and $H$. meleagridis as described above in: Identification of differentially abundant protein spots by MALDI-TOF/TOF. Mass tolerance in MS mode was set with 0.05 and 0.1 $\mathrm{Da}$ in MSMS mode for the rapid recalibration search, and $0.0011 \mathrm{Da}$ in $\mathrm{MS}$ and $0.01 \mathrm{Da}$ in MSMS mode for the final search. Parameters for database search: trypsin digestion, cysteine alkylation set to iodoacetamide, search effort set to rapid ID. FDR was performed using the integrated tools in ProteinPilot. Global FDR was set to $<1 \%$ on protein as well as on peptide level. Following the generally applied so-called "two-peptide rule" only proteins with at least two peptides were further considered as identified (Taylor and Goodlett, 2005).

\section{Shotgun Protein Identification Using LC-MS/MS}

For identification of the $H$. meleagridis proteins by LCMS/MS without previous gel electrophoretic separation, protein digestion was performed using a two-step in-solution digestion protocol with a combination of $\mathrm{Lys} C$ and trypsin according to the manufacturer's recommendation ${ }^{4}$ ). For each sample $10 \mu \mathrm{g}$ of total protein were diluted with $8 \mathrm{M}$ urea in $50 \mathrm{mM}$ Tris $\mathrm{pH} 8.0$, to a total volume of $10 \mu \mathrm{l}$. Proteins were reduced with $50 \mathrm{mM}$ DTT $\left(30 \mathrm{~min}, 37^{\circ} \mathrm{C}\right)$ and alkylated with $200 \mathrm{mM}$ IAA $\left(30 \mathrm{~min}, 25^{\circ} \mathrm{C}\right)$ before stepwise digestion with Trypsin/LysC mix (Promega) $(4 \mathrm{~h}$, $37^{\circ} \mathrm{C}$; dilution with $50 \mathrm{mM}$ Tris; $8 \mathrm{~h}, 37^{\circ} \mathrm{C}$ ). After acidification

\footnotetext{
${ }^{4}$ https://www.promega.com/-/media/files/resources/protocols/technical-
} manuals/101/trypsinlysc-mix-mass-spec-grade- protocol.pdf?la=en 
with concentrated TFA, samples were frozen at $-20^{\circ} \mathrm{C}$ until mass spectrometric analysis. An aliquot of peptides was analyzed by LC-MS/MS as described above in the section Identification of Differentially Abundant Protein Spots by LC-MS/MS.

\section{Label-Free Quantification and Statistical Analysis With SWATH MS Technology-Creation of SWATH Ion Library}

LC parameters for data independent SWATH quantification analyses were the same as described above for IDA injections. MS parameters were adapted for SWATH. MS1 spectra were collected in the range of $400-1,500 \mathrm{~m} / \mathrm{z}$ with an accumulation time of $50 \mathrm{~ms}$. Product ion spectra were collected in 34 windows in the range of $400-1,250 \mathrm{~m} / \mathrm{z}$ with a fixed width of 25 Da. For each window ions were accumulated for $80 \mathrm{~ms}$.

IDA identification results (see section Shotgun Protein Identification Using LC-MS/MS) were used to create the SWATH ion library with SWATH Acquisition MicroApp 2.0 in PeakView 2.2 (both Sciex, USA). Peptides were chosen based on a FDR rate $<1 \%$, excluding shared and modified peptides. Up to 6 peptides per protein and up to 6 transitions per peptide were used. Calculation of peak areas of SWATH samples was performed with MarkerView 1.2.1 (Sciex, USA) after retention time alignment and normalization using total area sums.

Further statistical evaluations were based on the normalized peak areas. Two different software tools were used: $\mathrm{R}$ programming language (R Development Core Team, 2014) as well as MarkerView (Sciex).
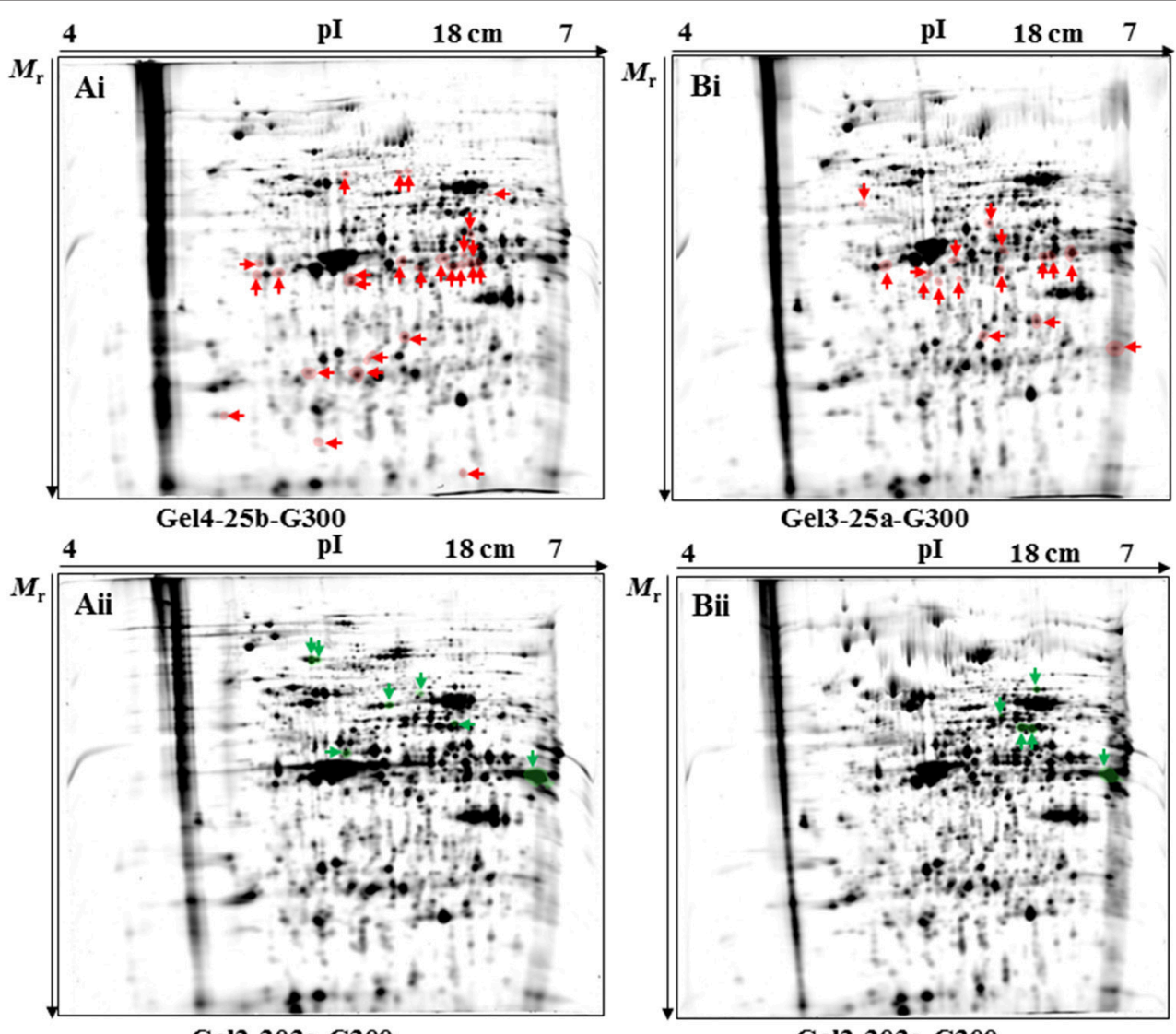

Gel2-303a-G300

FIGURE 1 | Position of protein spots detected as significantly $(P<0.05)$ differentially expressed by the 2D-DIGE- $\alpha$ (A) and 2D-DIGE- $\beta$ (B) experiment. Protein spots found to be significantly upregulated in the fluorescent images of the cultivated virulent strain (passage number 25) by the 2D-DIGE- $\alpha$ (Ai) and 2D-DIGE- $\beta$ (Bi) experiment are highlighted with red color and are indicated by red arrows. Fluorescent gel images with codenames Gel4-25b-G300 (Ai) and Gel3-25a-G300 (Bi) were chosen as the representative gel images for spot visualization. Protein spots found to be significantly upregulated in the fluorescent images of the cultivated attenuated strain (passage number 303) by the 2D-DIGE- $\alpha$ (Aii) and 2D-DIGE- $\beta$ (Bii) experiment are highlighted with green color and are indicated by green arrows. Fluorescent gels images with the codenames Gel2-303a-G300 (Aii) and Gel2-303a-G300 (Bii) were chosen as the representative gel images for spot visualization. The position of identified protein spots is additionally displayed on the corresponding silver-stained fluorescent gels (Figures S6, S7). The arrows at the $x$-and $y$ - axis in each gel are indicating the direction of protein mobility according to their pl (x-axis) and $M_{r}$ (y-axis). 2D-DIGE, two-dimensional differential gel electrophoresis; 25, virulent H. meleagridis parasites harvested at passage number 25; 303, attenuated $H$. meleagridis parasites harvested at passage number 303; $25 \mathrm{a}$ and $25 \mathrm{~b}$, protein samples extracted from virulent $H$. meleagridis parasites on day 1 and day 2 , respectively; 303a and 303b, protein samples extracted from attenuated $H$. meleagridis parasites on day 1 and day 2, respectively; G300, protein samples labeled with G-Dye 300; $M_{r}$, molecular mass; pl, isolelectric point. 


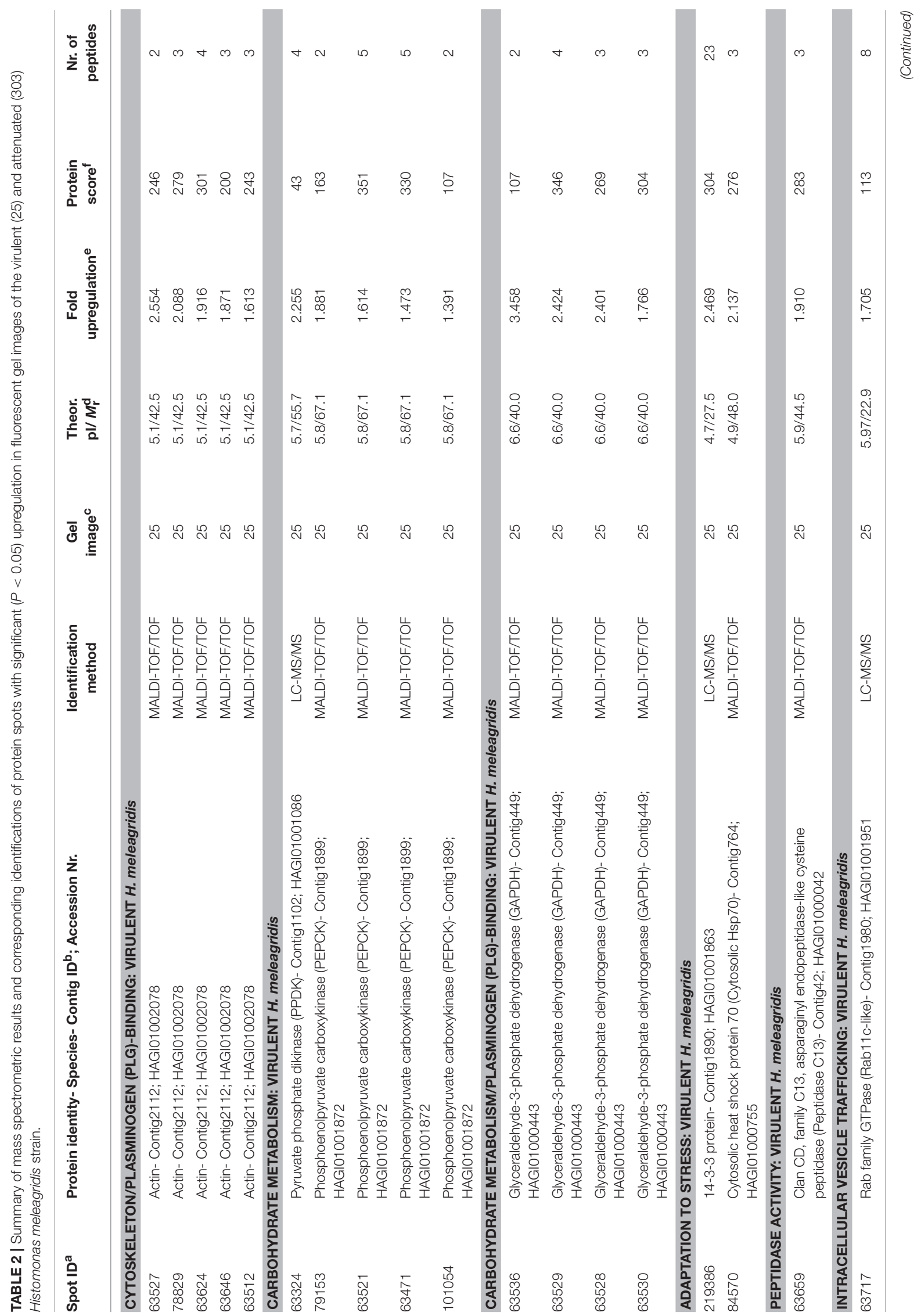




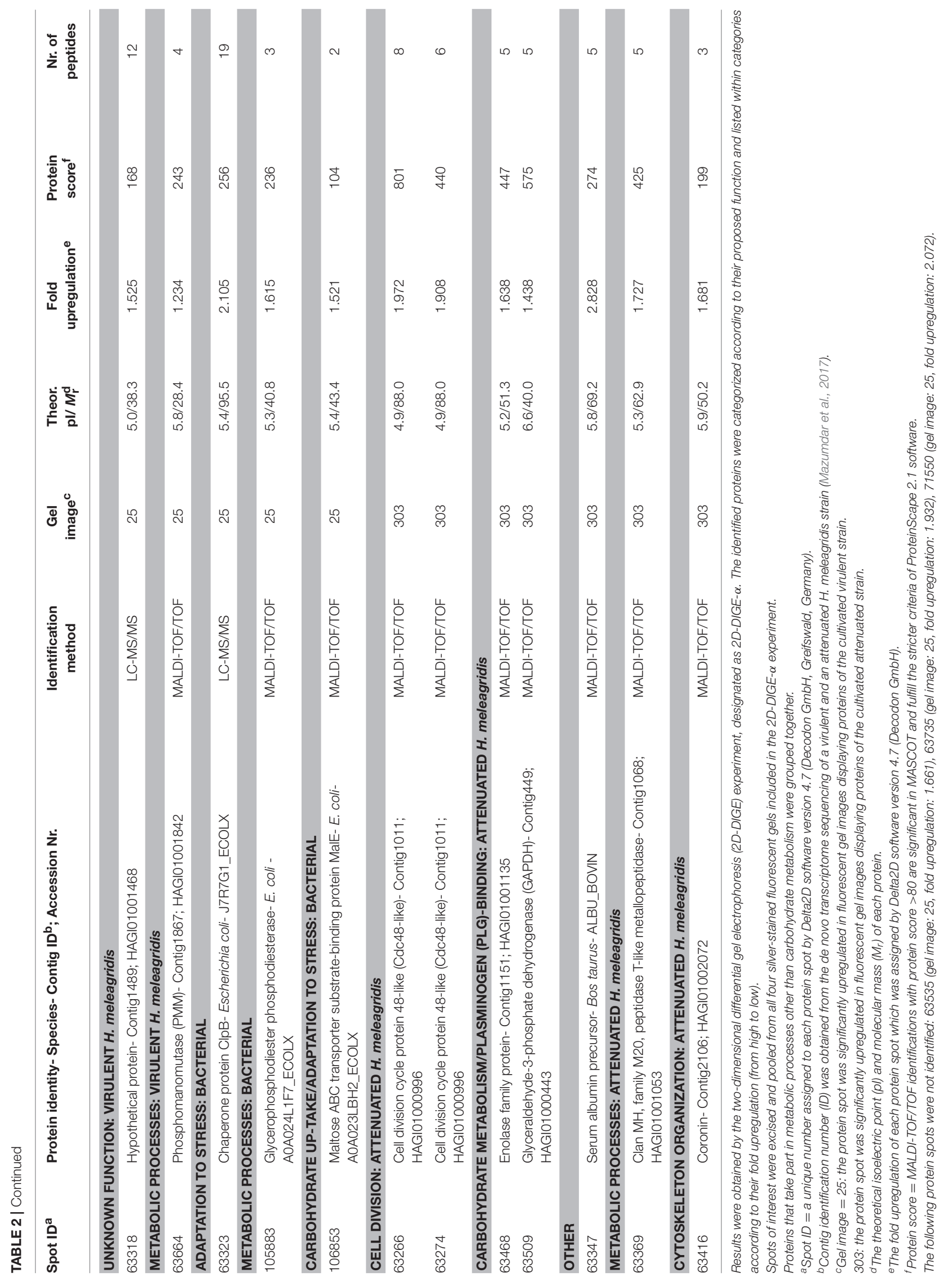




\section{Label-Free Quantification and Statistical Analysis With SWATH MS Technology-MarkerView}

After principal component analysis (PCA), groups were defined and a $t$-Test was performed. Proteins with at least two peptides were considered differential if the $p$-value was below $\alpha=0.00001$ and the absolute fold change was at least two (fold change $<$ -2 or $>+2$ ). As MarkerView does not differentiate between technical and biological replicates an additional statistical evaluation with $\mathrm{R}$ was performed.

\section{Label-Free Quantification and Statistical Analysis With SWATH MS Technology-R Programming Language}

cRAP proteins and proteins quantified with just one peptide were removed from the MarkerView normalized raw protein list before further processing. Raw peak areas were $\log 2$-transformed to approach a normal distribution. On a logarithmic scale, technical replicates were aggregated by arithmetic mean before application of statistical tests.

Differential expression of proteins was assessed using twotailed $t$-Test for independent samples for each protein. To adjust for multiple testing, the method of Benjamini and Hochberg was used to control the FDR (Benjamini and Hochberg, 1995). Protein expression was considered differential if the adjusted $p$ value was below $\alpha=0.08$ and the absolute fold change was at least two (fold change $<-2$ or $>+2$ ).

\section{Protein-Function Network Graphs Constructed for $\boldsymbol{H}$. meleagridis-Specific Proteins}

The open source software platform Cytoscape version 3.5.1 (Shannon et al., 2003) was used to display the link between identified H. meleagridis-specific proteins and their proposed function. The identified proteins were found to be significantly differentially expressed between the two strains by the $2 \mathrm{D}$ DIGE- $\alpha, 2$ D-DIGE- $\beta$, and SWATH MS experiments. The proposed functions were attributed to identified proteins based on their Gene Ontology terms, InterPro entries and existing literature. The fold upregulation values, which were acquired from Delta2D software version 4.7 (Decodon $\mathrm{GmbH}$ ) and SWATH MS quantification measurements, were mapped to certain colors. In this way, the $H$. meleagridis-specific proteins, which were represented by source nodes, were visually characterized and their level of expression was indicated.

\section{RESULTS}

\section{Fluorescent Protein Patterns of the in vitro Cultivated Virulent and Attenuated \\ H. meleagridis Parasites}

Protein spots were distributed within a $\mathrm{pH}$ range of 5-7 and a $M_{\mathrm{r}}$ of $15-140 \mathrm{kDa}$ (Figures S3, S4). The landmark spot, which was distinctive at $\mathrm{pI} 5$ and a $M_{\mathrm{r}}$ of $\sim 50 \mathrm{kDa}$ (Figures S3, S4), was known to be actin of $H$. meleagridis origin based upon a previous study (Monoyios et al., 2018). The visualization of this actin spot with yellow color was an indication that it was not differentially expressed between the in vitro cultivated virulent and attenuated parasites (Figure S3). This observation was later on confirmed by the statistical analysis of the fluorescent proteome patterns. The projection of virulent and attenuated protein spots with pseudocolors revealed that high $M_{\mathrm{r}}$ proteins $(50-140 \mathrm{kDa})$ were more abundant in the attenuated strain (Figures S3, S4). In contrast to this, proteins of low $M_{\mathrm{r}}(15-50 \mathrm{kDa})$ were more numerous in the protein samples of the virulent strain (Figures S3, S4).

\section{Detection of Significantly Differentially Abundant Protein Spots by 2D-DIGE}

Initially, 937 and 1297 protein spots were detected on the fusion images, which were generated by the 2D-DIGE- $\alpha$ and 2D-DIGE- $\beta$ computational image analysis, respectively. Artifacts and faint spots were removed by setting a spots' quality threshold and by performing manual spot editing. As a result, 563 and 762 protein spots were included in the statistical evaluation of fluorescent proteome patterns obtained by the 2D-DIGE- $\alpha$ and 2D-DIGE- $\beta$ experiment, respectively.

In the 2D-DIGE- $\alpha$ experiment, the statistical analysis detected a total number of 33 protein spots as differentially expressed (Figures 1Ai,Aii and Figure S5A). The fold change in their expression levels ranged between 1.234 and 3.458 (Table 2). Twenty-six out of the 33 protein spots were significantly overexpressed in gel images containing proteins of the virulent culture (Figure 1Ai). However, three out of these could not be located on silver-stained fluorescent gels obtained by the 2D-DIGE- $\alpha$ experiment (Figure S6A). The seven remaining protein spots, out of the total 33, were found overexpressed in the gel images that contained proteins of the attenuated culture (Figure 1Aii). All seven spots were located at a $M_{\mathrm{r}}$ of $50 \mathrm{kDa}$ and above (Figure 1Aii, Figure S6B). In contrast, only four out of the 26 protein spots, with overexpression in the gel images of the virulent culture, were located above $50 \mathrm{kDa}$ (Figure 1Ai Figure $66 \mathrm{~A}$ ).

In the 2D-DIGE- $\beta$ experiment the statistical analysis detected a total number of 21 protein spots as differentially expressed (Figures 1Bi,Bii and Figure S5B). The fold change in their expression levels ranged between 1.255 and 3.443 (Table 3). Sixteen out of the 21 differentially expressed protein spots were detected in gel images containing proteins of the virulent culture (Figure 1Bi). Identifying two out of these spots from silver-stained fluorescent gels was not possible (Figure S7A). In agreement with the 2D-DIGE- $\alpha$ experiment, only two out of the 16 protein spots were located above $50 \mathrm{kDa}$ (Figure 1Bi, Figure S7A). The five remaining spots, out of the total 21, were detected in the gel images of the attenuated culture (Figure 1Bii). As it was observed in the 2D-DIGE- $\alpha$ experiment for this strain, all five spots were located at a $M_{\mathrm{r}}$ of $\geq 50 \mathrm{kDa}$ (Figure 1Bii, Figure S7B). 


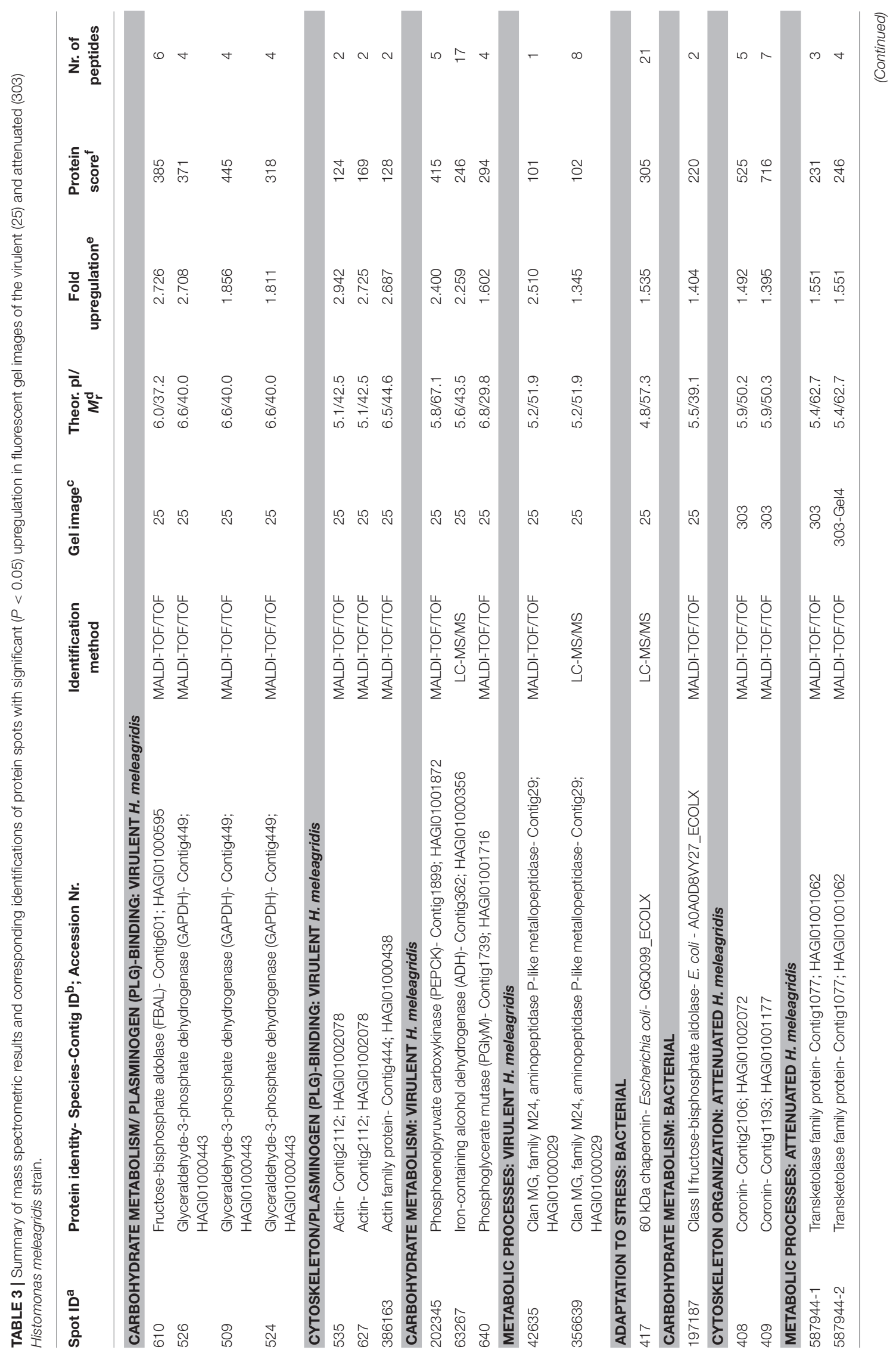




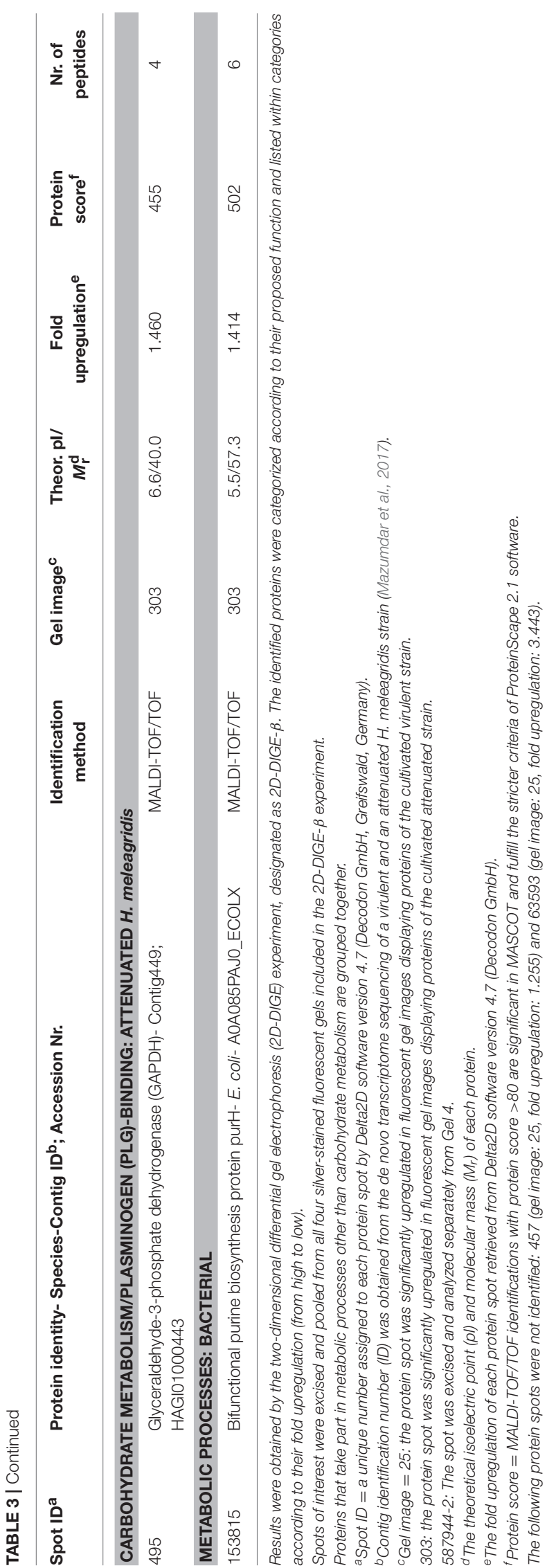

\section{Identification of Proteins Detected as Significantly Differentially Abundant by the 2D-DIGE Experiments}

Thirty and 19 protein spots, which were detected as significantly $(P<0.05)$ overexpressed by the 2D-DIGE- $\alpha$ and 2D-DIGE$\beta$ analysis, respectively, matched with entries from the aforementioned databases (see the section Identification of Differentially Abundant Protein Spots by MALDI-TOF/TOF) (Tables 2, 3 and Tables S1, S2). In the 2D-DIGE- $\alpha$ experiment the 23 spots, which displayed upregulation in the gel images of the virulent culture, were shown to correspond to 13 different proteins (Table 2, Figure 2A), while in the 2DDIGE- $\beta$ experiment the 14 protein spots from the same culture were identified as 10 unique proteins (Table 3, Figure 2A). Between the two experiments the common proteins, with overexpression in the virulent strain, were: actin, glyceraldehyde 3-phosphate dehydrogenase (GAPDH) and phosphoenolpyruvate carboxykinase (PEPCK) (Tables 2, 3 and Figure 2A). In the 2D-DIGE- $\alpha$ experiment, the seven upregulated protein spots from the attenuated culture, were identified as six different proteins (Table 2, Figure 2B), while in the 2D-DIGE- $\beta$ experiment the five protein spots, which exhibited overexpression in the fluorescent gel images of the same culture, represented four unique proteins (Table 3, Figure 2B). In the attenuated culture, the common unique proteins between the two experiments were: coronin and GAPDH (Tables 2, 3 and Figure 2B).

\section{Identification of Protein Spots Detected as Significantly Overexpressed in Fluorescent Gel Images of the Virulent $\boldsymbol{H}$. meleagridis Culture}

In the 2D-DIGE- $\alpha$ experiment 20 out of the 23 identified protein spots, with overexpression in the virulent culture, corresponded to $10 \mathrm{H}$. meleagridis-specific proteins (Table 2). Based on their proposed functions these identified proteins can be attributed to eight different categories (Figure 3A). The most frequent identified proteins were actin, followed by GAPDH and PEPCK (Table 2, Figure 3A). As shown by the color-coded source nodes, the protein spots with the highest upregulation were identified as GAPDH $(63528,63529,63536)$, actin (63527), and 14-3-3 protein (219386) (Table 2, Figure 3A). Nine identified protein spots corresponded to two unique proteins, namely actin, represented by five spots $(63512,63624,63527,63646,78829)$ and GAPDH, represented by four spots $(63528,63529,63530,63536)$ which might be associated with alternative functions (Figure 3A). The four GAPDH spots, which were aligned at a $M_{\mathrm{r}}$ of $\sim 40 \mathrm{kDa}$, exhibited slight differences in their $\mathrm{pI}$ but were all mapped to contig449 (Table 2 and Figures S8Ai,Aii). The five actin spots demonstrated shifts in their $\mathrm{pI}$ and were dispersed at the low $M_{\mathrm{r}}$ area between 25 and $50 \mathrm{kDa}$ (Figures S8Ai,Aii). All actin spots could be mapped to contig2112 (Table 2). The statistical analysis, clustered the actin spots together with a Rab11 isotype (cluster 1), heat shock protein 70 (Hsp70) (cluster 3), legumainlike cysteine peptidase (CP) (cluster 4), and the four GAPDH protein spots (cluster 5) (Figure S5A). The clustering indicates 
A 2D-DIGE- $\alpha$ (Vir)

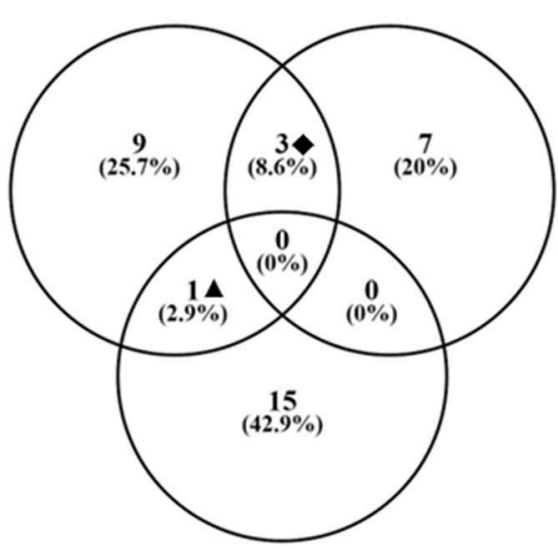

SWATH MS (Vir)

\section{B 2D-DIGE- $\alpha$ (Atte) 2D-DIGE- $\beta$ (Atte)}

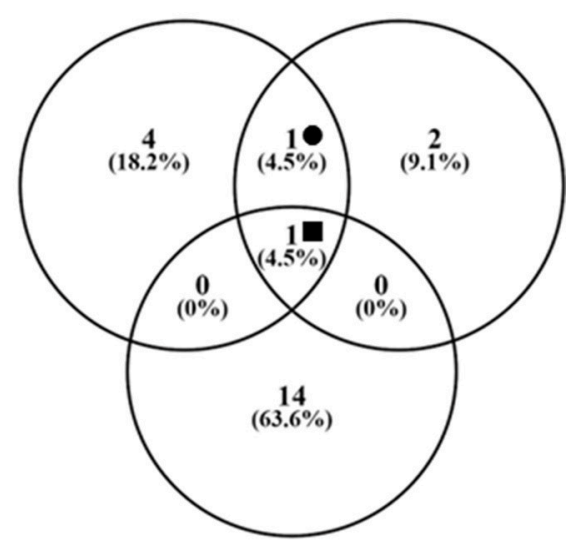

SWATH MS (Atte)

FIGURE 2 | Number of unique and shared identifications for proteins detected as significantly upregulated in the virulent (A) and attenuated (B) cultivated $H$. meleagridis strain by the 2D-DIGE- $\alpha, 2 \mathrm{D}-\mathrm{DIGE}-\beta$, and SWATH MS experiments. Venny 2.1.0 tool (Oliveros, 2015) was used to summarize the findings with venn diagrams. The same extracted protein samples were analyzed by the 2D-DIGE- $\alpha$ and SWATH MS experiment. Based on the analyses the following identified proteins were found to be common between experiments: $=$ actin, GAPDH and PEPCK (A); $\boldsymbol{\Delta}=$ Clan CD, family C13, asparaginyl endopeptidase-like CP (A); $\bullet=$ GAPDH (B); = Coronin (B). 2D-DIGE, two-dimensional differential gel electrophoresis; CP, cysteine peptidase; SWATH MS, sequential window acquisition of all theoretical mass spectra mass spectrometry; GAPDH, glyceraldehyde-3-phosphate dehydrogenase; PEPCK, phosphoenolpyruvate carboxykinase.

co-regulation of the above proteins. The remaining three spots, found to be overexpressed in the gel images of the virulent culture, represented three proteins of $E$. coli origin that seem to be associated with metabolism and adaptation to stress (Table 2).

In the $2 \mathrm{D}$-DIGE- $\beta$ group 12 out of the 14 identified protein spots, with upregulation in the gel images of the virulent culture, represented eight unique $H$. meleagridis proteins (Table 3). Based on their proposed functions, the $H$. meleagridis-specific proteins could be connected with four different categories (Figure 3B). The most frequent identified proteins were GAPDH, in three spots out of the total $12(509,524,526)$, followed by actin, which was represented by two spots $(535,627)$ (Table 3, Figure 3B). According to the color-coded network, protein spots exhibiting the highest upregulation were identified as actin $(535,627)$, fructose-bisphosphate aldolase (FBAL) (610), GAPDH (526), and actin family protein (386163) (Table 3 , Figure $3 B$ ). It should be noted that eight out of the $12 \mathrm{H}$. meleagridis-specific protein spots displayed an upregulation level, which was above the median value (Table 3, Figure 3B). Five out of the eight unique $H$. meleagridis proteins could be connected with carbohydrate metabolism (509, 524, 526, 610, 640, 63267, 202345) (Figure 3B). Six protein spots corresponded to three unique proteins, namely GAPDH (509, 524, 526), actin (535, 627), and FBAL (610) (Figure 3B). Although unrelated, they might be associated with alternative functions. In agreement with the 2D-DIGE- $\alpha$ experiment the three GAPDH protein spots, were also mapped to contig449 and were aligned at a $M_{\mathrm{r}}$ of $\sim 40 \mathrm{kDa}$ with minor differences in their pI (Table 3 and Figures S8Bi,Bii). The actin spot with identification number 627 , displayed a $M_{\mathrm{r}}$ which was $\sim 15 \mathrm{kDa}$ lower and a $\mathrm{pI}$ value which was approximately one unit larger in comparison to the 535 actin spot (Figures S8Bi,Bii). In the statistical analysis, these two actin spots $(535,627)$ clustered with a GAPDH spot (509), the FBAL spot (610), a Clan MG, family M24, aminopeptidase P-like metallopeptidase (42635), and with other proteins of the carbohydrate metabolism, namely PEPCK (202345) and phosphoglycerate mutase (PGlyM) (640) (cluster 3, Figure S5B). Additionally, another GAPDH spot (524) clustered with an actin family protein (386163) (cluster 1, Figure S5B). The remaining two spots originated from the bacterial background and were connected with stress adaptation and carbohydrate metabolism (Table 3).

\section{Identification of Protein Spots Detected as Significantly Upregulated in Fluorescent Gel Images of the Attenuated H. meleagridis Culture}

In the 2D-DIGE- $\alpha$ experiment, six out of the seven analyzed spots, which were upregulated in the gel images of the attenuated culture, corresponded to five proteins of the protozoan (Table 2). These proteins can be connected with five different categories (Figure 4A). As demonstrated by the protein network, the protein spots with the highest upregulation were identified as a Cdc48-like protein $(63266,63274)$ and a Clan MH, family M20, peptidase T-like metallopeptidase (63369) (Table 2, Figure 4A). The two Cdc48-like protein spots were found to be closely associated at a $M_{\mathrm{r}}$ of $\sim 110 \mathrm{kDa}$ (Figures S9Ai,Aii). In contrast to the virulent strain, GAPDH was identified in a single significantly upregulated spot (63509), which was located at a position that corresponded to its calculated pI (Table 2 and Figures S9Ai,Aii). The same single GAPDH spot was also visible in the gel images of the virulent culture at the same $\mathrm{pI}$ value (Figures S8Ai,Aii), albeit 

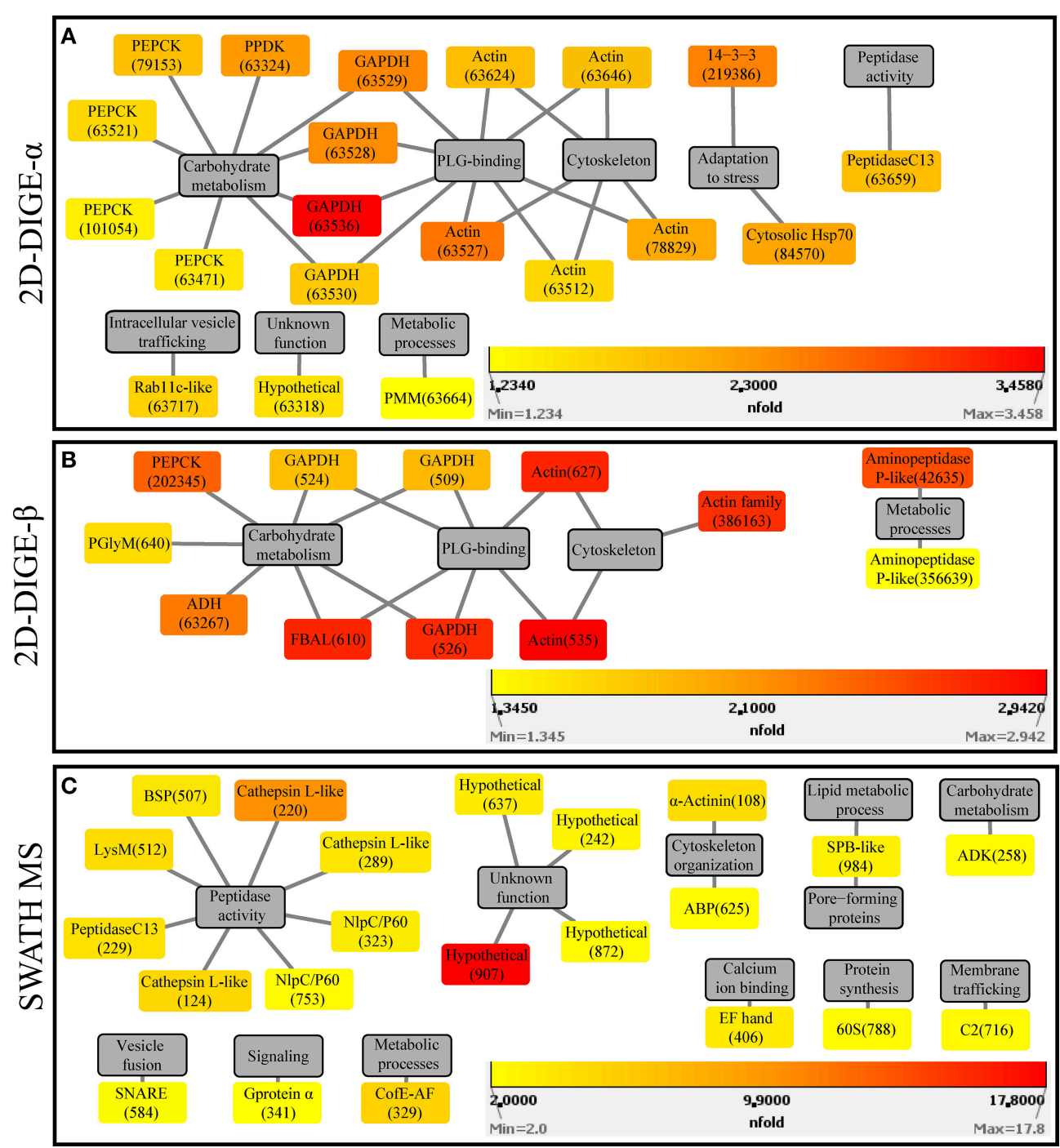

FIGURE 3 | Protein-function network graphs of Histomonas meleagridis-specific proteins detected as significantly overexpressed in the virulent strain by the 2D-DIGE- $\alpha$ (A), 2D-DIGE- $\beta$ (B), and SWATH MS (C) experiments. The protein identifications are displayed as color-coded source nodes, with their edges connected to gray colored target nodes which represent the proposed functions. The numbers in parentheses represent the identification numbers assigned to each protein by Delta2D software version 4.7 (Decodon GmbH, Greifswald, Germany) and SWATH MS experiment. In each panel, the dialogs on the right lower corners are the yellow-orange-red gradient which was selected to represent the fold upregulation values of overexpressed proteins from the virulent strain. The source nodes were color-coded based on the fold upregulation data associated with each significantly differentially expressed protein (Tables 2-4). The network layout was manually determined. The reader is referred to Tables 2-4 for explanation of abbreviated protein names. 2D-DIGE, two-dimensional differential gel electrophoresis; SWATH MS, sequential window acquisition of all theoretical mass spectra mass spectrometry.

in the attenuated strain it exhibited a slightly higher upregulation (Table 2). The only spot that did not belong to the protozoan was identified as bovine serum albumin (Table 2). This spot exhibited the highest upregulation in the gel images of the attenuated strain but its presence originated from the culture medium (Table 2).

In the 2 D-DIGE- $\beta$ group, four out of five spots, which demonstrated upregulation in the gel images of the attenuated culture, represented three proteins of $H$. meleagridis (Table 3). These proteins can be associated with four different categories (Figure 4B). As shown in the protein network, the protein spots with the highest upregulation were a transketolase family protein
(587944) and coronin (408) (Table 3 and Figure 4B). Coronin was also identified in a second spot (409) but was mapped to a different contig (Table 3 ). The two coronin spots displayed similar fold upregulation values and were visible, as a pair, at a position that corresponded to their correct pI but at $\sim 20$ $\mathrm{kDa}$ higher than their calculated $M_{\mathrm{r}}$ (Figures S9Bi,Bii). The same prominent GAPDH protein spot (495), which displayed a significant upregulation in the 2D-DIGE- $\alpha$ experiment, was also detected as overexpressed here at a position that corresponded to its correct pI (Table 3 and Figures S9Bi,Bii). Due to its size and position, this spot was distinguishable from its post-translational 

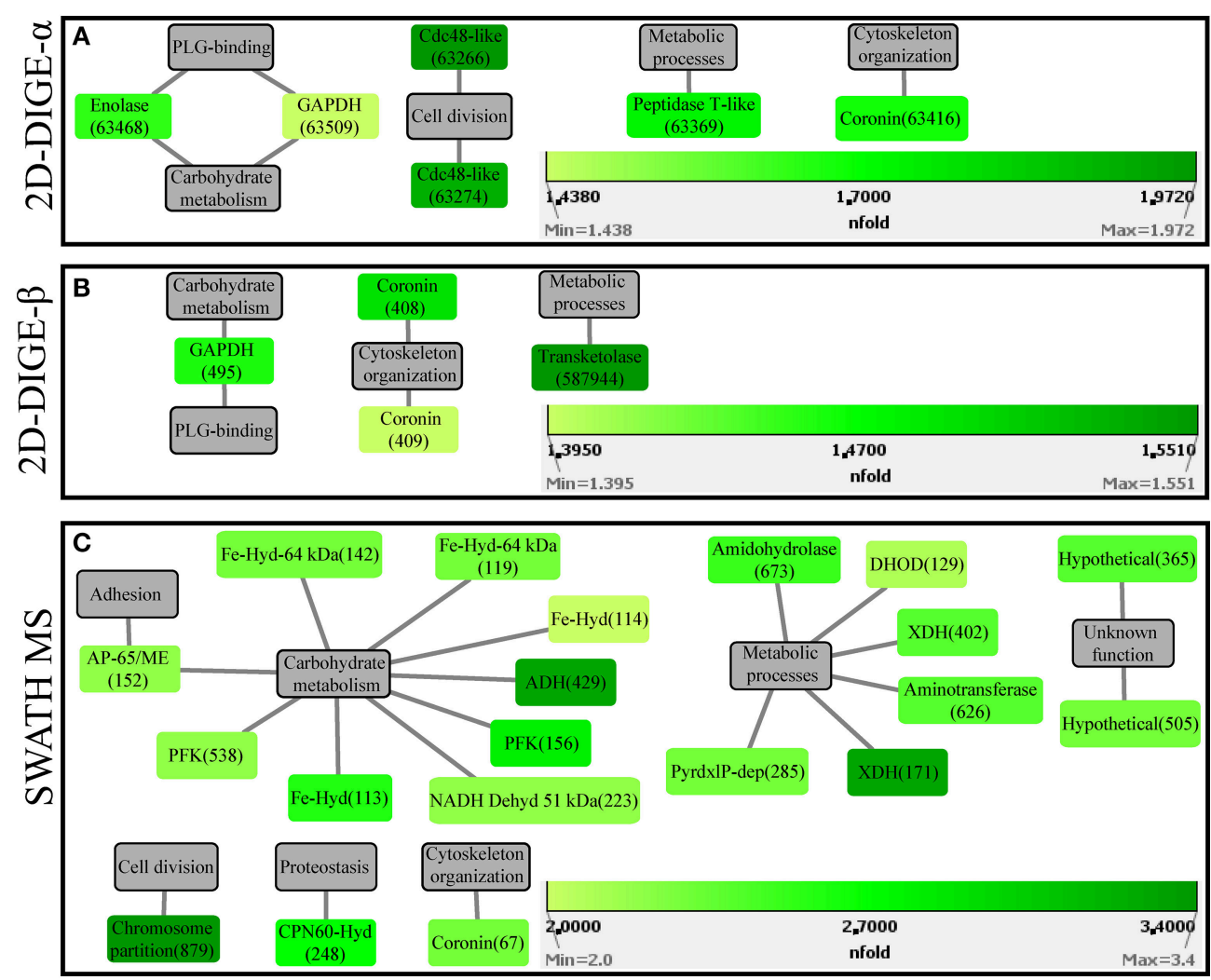

FIGURE 4 | Protein-function network graphs of Histomonas meleagridis-specific proteins detected as significantly overexpressed in the attenuated strain by the 2D-DIGE- $\alpha$ (A), 2D-DIGE- $\beta$ (B), and SWATH MS (C) experiments. The protein identifications are displayed as color-coded source nodes, with their edges connected to gray colored target nodes which represent the proposed functions. The numbers in parentheses represent the identification numbers assigned to each protein by Delta2D software version 4.7 (Decodon GmbH, Greifswald, Germany) and SWATH MS experiment. In each panel, the dialogs on the right lower corners are the green colored gradient which was selected to represent the fold upregulation values of overexpressed proteins from the attenuated strain. The source nodes were color-coded based on the fold upregulation data associated with each significantly differentially expressed protein (Tables 2-4). The network layout was manually determined. The reader is referred to Tables 2-4 for explanation of abbreviated protein names. 2D-DIGE, two-dimensional differential gel electrophoresis; SWATH MS, sequential window acquisition of all theoretical mass spectra mass spectrometry.

modifications which were upregulated in the gel images of the virulent culture (Figures S8Bi,Bii). In contrast with the findings of the 2D-DIGE- $\alpha$ experiment for the attenuated strain, one overexpressed spot exhibited homology with an E. coli protein and was associated with purine metabolism (Table 3).

\section{Identification of Proteins Detected as Significantly Differentially Regulated by SWATH MS}

An average number of 832 and 878 proteins were identified in the protein samples which were obtained from the virulent (25a and 25b) and attenuated (303a and 303b) H. meleagridis culture, respectively. The statistical analysis detected 42 significantly differentially expressed proteins of which four proteins fulfilled the significance criteria of MarkerView, 10 proteins fulfilled the criteria of $\mathrm{R}$ programming language and 28 proteins were detected as statistical significant by both methods (Table 4). From these differentially regulated proteins, 22 originated from the virulent and 20 from the attenuated $H$. meleagridis culture (Table 4, Table S3).

The fold change in upregulation values ranged between 2.0 and 17.8 for proteins of the virulent strain and between 2.0 and 3.4 for proteins of the attenuated one (Table 4 and Figures 3C, 4C). The 22 upregulated proteins in the virulent culture corresponded to 16 different proteins, one of which was also found to be significantly overexpressed in the $2 \mathrm{D}$ DIGE- $\alpha$ experiments and identified as Clan CD, family C13, asparaginyl endopeptidase-like CP (Figure 2A). Based on their proposed functions, the 22 proteins could be connected with 12 different categories (Figure 3C). Peptidase activity was the largest category, which was represented by eight identifications each mapped to a different contig (Table 4, Figure 3C). The most frequent identification was a group of four proteins with unknown function termed hypothetical, followed by clan CA, family C1, cathepsin L-like CP which was represented by three proteins (Table 4, Figure 3C). The four hypothetical proteins were encoded by different contigs and that was also the case for the three proteins identified as cathepsin L-like CP (Table 4). 


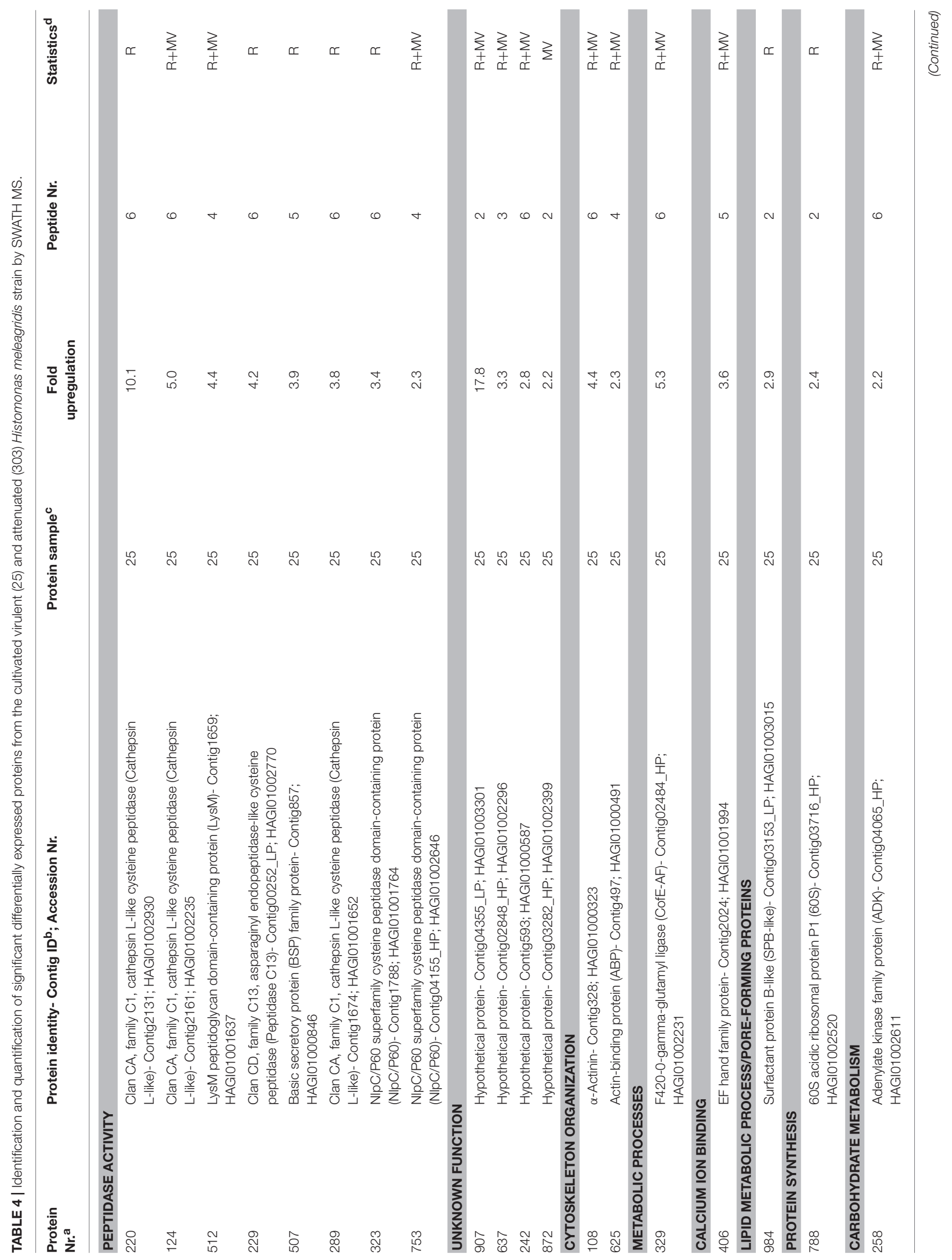




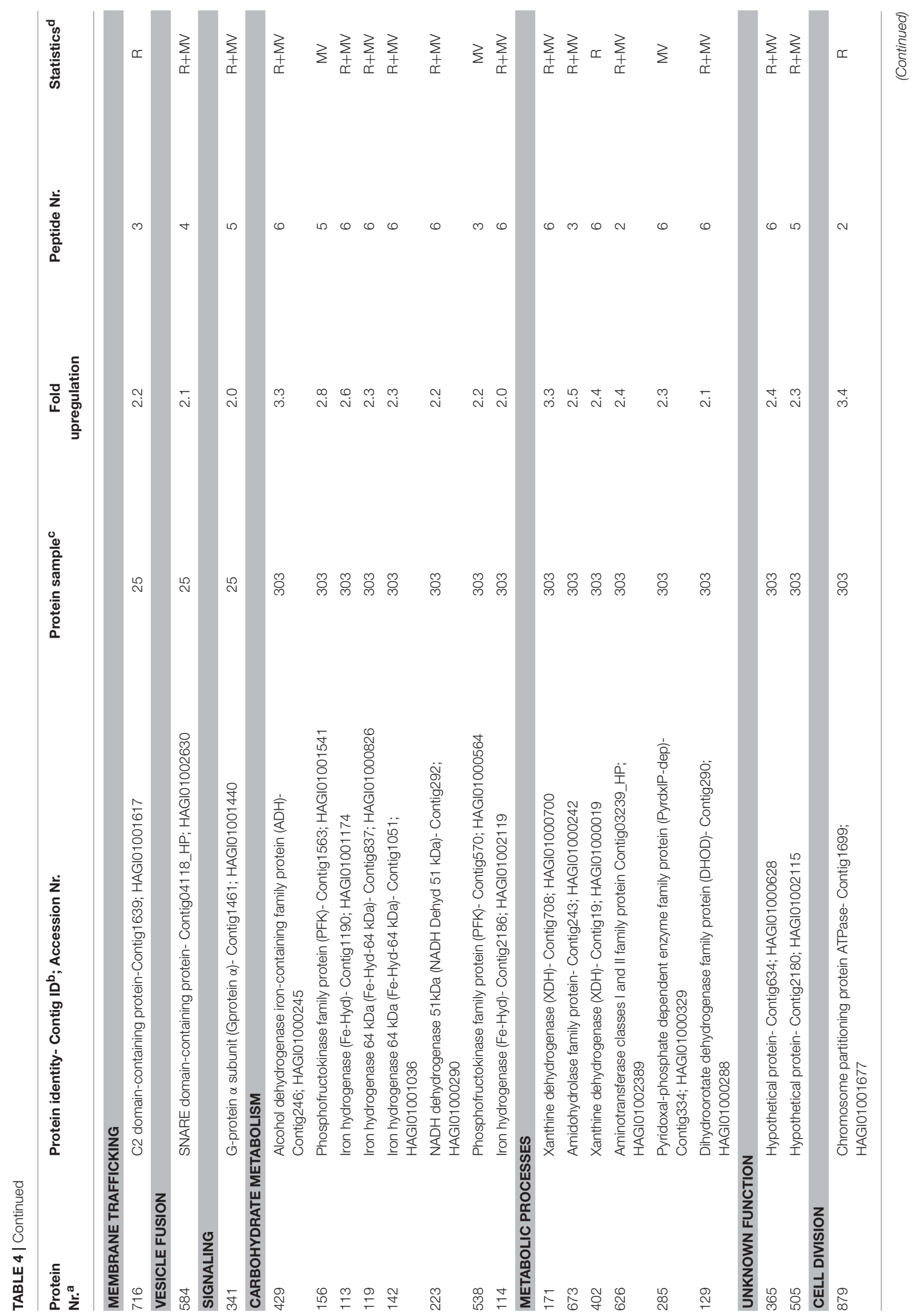




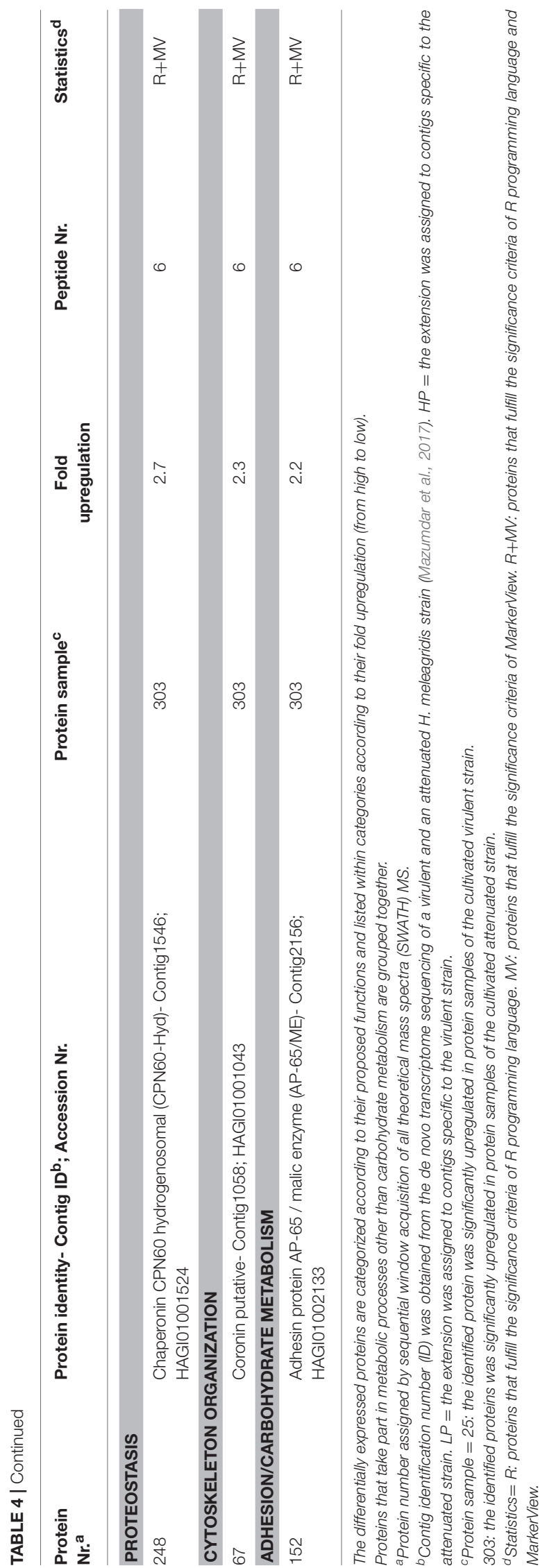

Proteins with the highest upregulation were a hypothetical protein (907) and a clan CA, family C1, cathepsin L-like CP (220). In this strain, most of the identified proteins displayed a mediocre fold upregulation level which was below the median value (Table 4).

The 20 overexpressed proteins in samples of the attenuated culture represented 15 different proteins, out of which only coronin was shown to be also significantly upregulated in the gel-based experiments (Table 4, Figure 2B). The upregulated coronin protein, reported by SWATH MS, was mapped to a contig which was different from the one to which the coronins, reported by the 2D-DIGE experiments, were mapped (Tables 24). The 20 overexpressed proteins could be connected with seven different categories based on their proposed functions (Figure 4C). Carbohydrate metabolism constituted the largest category, which was represented by nine identifications each mapped to a different contig (Table 4, Figure 4C). Within this group, iron hydrogenases were the most frequently identified proteins (Figure 4C). The four iron hydrogenases were encoded by different contigs and could be separated into two groups based on their annotation (Table 4). The second largest category was a group of six proteins that are involved in other metabolic processes (Figure 4C). The range of fold upregulation values for these 20 proteins was narrower in comparison to the one reported for the virulent strain (Table 4). Nevertheless, as for the virulent strain, most of the 20 proteins from the attenuated one demonstrated mediocre upregulation which was below the median value (Table 4, Figure 4C). Proteins with the highest upregulation belonged to categories such as cell division (879), carbohydrate metabolism (429), and metabolic processes (171) (Table 4, Figure 4C).

In contrast to the gel-based experiments, none of the above 42 significantly differentially expressed proteins belonged to $E$. coli $\mathrm{DH} 5 \alpha$. However, an average number of 151 and 158 proteins were identified to be of bacterial origin in protein samples obtained from the in vitro cultivated virulent (25a and 25b) and attenuated (303a and 303b) strains, respectively.

\section{DISCUSSION}

\section{2-DE and 2D-DIGE Experiments}

Despite substantial differences between 2-DE and 2D-DIGE, such as in the experimental design, the applied amount of protein, influence of technical variation and protein spot detection, the protein patterns obtained by the two techniques showed a high level of similarity. The observed distribution of protein spots in the 2D-DIGE- $\alpha$ and 2D-DIGE- $\beta$ experiments was in agreement with previously published conventional 2-DE gels of $H$. meleagridis cultures (Monoyios et al., 2018). In the conventional 2-DE study as well as in the 2D-DIGE, a noticeable difference in the $M_{\mathrm{r}}$ distribution of proteins between virulent and attenuated culture samples was revealed (Monoyios et al., 2018). In particular, low $M_{\mathrm{r}}$ proteins were more numerous in the gel images of the virulent strain, while high $M_{\mathrm{r}}$ proteins were more abundant in the gel images of the attenuated one. This could be attributed to a stronger proteolytic activity that might have occurred within the virulent $H$. meleagridis parasites. 
Evidence for this, could be found in the shotgun proteomic analysis, in which a number of CPs were found to be significantly upregulated in protein samples of the virulent strain, one of which exhibited the second highest upregulation value among all others.

A number of proteins which were found to be significantly overexpressed in the 2-DE study were re-detected in the two 2DDIGE experiments, a finding that underscores their importance. Interestingly, these proteins were also mapped to the same contigs. In the virulent strain proteins such as 14-3-3, Hsp70, multiple actin proteins of low $M_{\mathrm{r}}$ and the legumain-like CP were re-discovered as significantly upregulated. The latter protein was also detected as upregulated in the same strain by the SWATH MS experiment but was mapped to a different contig. Nevertheless, knowing that this CP was consistently detected in the virulent strain by three independent proteomic experiments verifies its implication in virulence mechanisms. On the other hand, in the fluorescent gel images of the attenuated strain, the Cdc48-like protein, enolase, and coronin were re-detected. Coronin, a protein implicated in actin cytoskeleton remodeling, was also found overexpressed in the attenuated strain by the SWATH MS experiment but mapped to a different contig.

\section{D-DIGE and SWATH MS Experiments}

Notwithstanding their utility, gel-based techniques cannot visualize complete proteomes (reviewed in Rabilloud et al., 2010). In a given 2-DE gel only specific proteins can be visualized depending on a number of factors such as: solubility, pI, relative abundance, $M_{\mathrm{r}}$, gel size, and $\mathrm{pH}$ gradient used (Görg et al., 2004; reviewed in Baggerman et al., 2005). The utilization of a gelfree, label-free shotgun proteomic approach with SWATH MS technology complements the 2D-DIGE gel-based experiments, since the resolution and detection of significant proteins is not depended on the $\mathrm{pH}$ gradient of the IPG strip, the length of the IPG strip or the polyacrylamide gel. A characteristic observed in our study as well. However, in spite of their resolving power, shotgun proteomic methods are preoccupied with the analysis of peptides and cannot conclude if a given identified protein was subjected to proteolysis or post-translational modifications as information on their experimental pI and $M_{\mathrm{r}}$ is lost (Görg et al., 2004). This feature was observed in the case of actin and GAPDH identifications, whose multiple spots were found upregulated in the virulent strain in both 2D-DIGE experiments, but SWATH analysis missed to detect them.

\section{Proteins With Plasminogen and Fibronectin-Binding Potential}

Although they are traditionally connected with non-virulent mechanisms, proteins such as actin, GAPDH and FBAL demonstrated the highest upregulation in fluorescent gel images of the virulent strain. Actin is an essential component of cytoskeleton with involvement in cell motility and cellular shape maintenance (reviewed in Dominguez and Holmes, 2011). In anaerobic protozoa such as Trichomonas vaginalis, GAPDH and FBAL participate in the glycolytic part of carbohydrate metabolism, which takes place in the cytoplasm (Huang et al., 2014).
The upregulated actin and GAPDH proteins displayed $\mathrm{pI}$ and $M_{\mathrm{r}}$ values, which were different from those displayed by the prominent actin and GAPDH proteins that were visualized on the same gels. Interestingly, regardless of their gel position, the actin and GAPDH proteins were all mapped to the same contigs. This finding supports the view that these multiple post-translationally modified or truncated proteins might be components of other mechanisms which are not related with cytoskeleton or carbohydrate metabolism. The experiments of Lama and coworkers demonstrated that GAPDH is a fibronectin (FN)-, plasminogen (PLG)- and collagen-binding protein with localization on T. vaginalis surface (Lama et al., 2009). Insoluble FN is a large glycoprotein of the extracellular matrix (ECM) that connects, via integral membrane proteins known as integrins, cellular surfaces with collagen (Hynes and Yamada, 1982; Bachman et al., 2015). It was theorized that the association of T. vaginalis with ECM components can promote colonization of the host (Lama et al., 2009).

Common characteristics of actin, FBAL and GAPDH are their alternative localization on parasites' surface and the ability to bind PLG (Lama et al., 2009; González-Miguel et al., 2015). PLG binding to these surface proteins, is an important step for its activation to plasmin and for maintaining its activation (reviewed in Plow et al., 1995). Lysine residues that are externally localized in PLG surface receptors are usually implicated in PLG binding (González-Miguel et al., 2015). Interestingly, in $H$. meleagridis actin the presence of a domain with similarities to a proposed PLG-binding domain of other organisms was discussed (González-Miguel et al., 2015; Monoyios et al., 2018). Plasmin degrades ECM and can help parasites that reside in blood vessels such as Dirofilaria immitis, to avoid blood clots due to its fibrinolytic activity (González-Miguel et al., 2015). Considering that $H$. meleagridis can spread via blood vessels to the liver and to other organs during the final stages of the disease (Singh et al., 2008), the ability to bind PLG might be of equal importance for this flagellate.

\section{Cytolytic Factors}

The SWATH MS experiment identified a pore-forming protein (PFP), namely surfactant protein B-like (SPB-like), being overexpressed in the virulent strain. SPB contains a motif of six cysteine periodically located residues, which is commonly found in proteins of the saposin-like family (reviewed in Hawgood et al., 1998). This saposin motif is also found in molecules with antibacterial activity such as the lysin of Natural Killer cells (NK-lysin) and the PFPs of Entamoeba histolytica, termed amoebapores (reviewed in Hawgood et al., 1998). In addition to structural similarities, the proteins mentioned above, demonstrate similar mode of action. This involves attachment to membranes that possess negatively charged phospholipids, following the lysis or fusion of such membranes (reviewed in Hawgood et al., 1998). Once PFPs penetrate the lipid bilayer of the target cell, death occurs by osmotic lysis (reviewed in Hirt et al., 2011). The activity of PFPs depends on the $\mathrm{pH}$ of the micro-space which is created between the parasite and the host's cell, following amoeboid transformation and cytoadherence (reviewed in Hirt et al., 2011). Based on the above, it is evident 
that SPB-like can be an effective virulence factor. Nevertheless, in the in vitro environment the protein might also contribute to the lysis of bacteria and through this to nutrient acquisition.

A clan CA, family C1, cathepsin L-like and a clan CD, family C13, asparaginyl endopeptidase-like CPs were significantly upregulated in the virulent low-passaged $H$. meleagridis parasite. In $T$. vaginalis almost half of the predicted peptidases contain cysteine as an active site similar to $H$. meleagridis transcriptome in which CPs were the most represented group of peptidases (reviewed in Hirt et al., 2011; Mazumdar et al., 2017). This category of peptidases participates in $T$. vaginalis virulence mechanisms such as cytoadherence, nutrient acquisition, hemolysis and apoptosis of human vaginal epithelial cells (reviewed in Hirt et al., 2011). Aside from this, the proteolytic activity of cathepsin L-like CPs, secreted by Trichomonas gallinae, was connected to the cytopathogenic effect on permanent chicken liver cells indicating their role in virulence of this avian parasite (Amin et al., 2012). The differential expression of cathepsin L-like CP was detected comparing 2DE gels of high- and low-virulence T. vaginalis isolates (De Jesus et al., 2009). However, in contrast to our finding, the T. vaginalis clan $\mathrm{CD}$, family $\mathrm{C} 13$, asparaginyl endopeptidase-like CP did not exhibit differential expression among these isolates (De Jesus et al., 2009). It was theorized that T. vaginalis CPs are involved in the lysis of erythrocytes and through this action can obtain lipids and iron, which cannot be synthesized otherwise (Rosset et al., 2002). Considering, that the in vitro growth of $H$. meleagridis can be significantly enhanced by the presence of supplemental cholesterol (Gruber et al., 2018) one cannot exclude the involvement of CPs in similar processes.

Rab11-isotypes, one of which was detected to be overexpressed in the virulent strain by $2 \mathrm{D}$-DIGE, could be implicated in the secretion of CPs. E. histolytica parasites which were overexpressing a Rab11 isotype, demonstrated augmentation in the amounts of intracellular and secreted CPs (Mitra et al., 2007). The overexpression of this isotype, was not connected with higher production of CPs, but rather with an increase in transportation (Mitra et al., 2007). While RabGTPases modulate the contact of membranes, SNARE proteins participate in the actual act of fusion and lipid bilayer mixing (Ungermann and Langosch, 2005). In this context, the detection of an overexpressed SNARE domain-containing protein in the virulent parasite could increase the fusion of $\mathrm{CP}$-containing vesicles with the plasma membrane and the release of CPs.

\section{Cytoadherence Mechanisms}

The SWATH experiment identified the $\alpha$ subunit of the heterotrimeric membrane-associated $G$ protein as upregulated in the virulent strain. The $G$ protein subunits can activate other molecules, including phospholipase C (InterPro entry ${ }^{5}$ ). The activity of phospholipase $\mathrm{C}$ on phospholipids can alter the negative surface charge of $T$. vaginalis, and as a result the interaction of this human parasite with the host's cells is initiated (reviewed in López et al., 2000). Additionally, the creation of cytoplasmic extensions such as pseudopodia and filopodia is

$\overline{{ }^{5} \text { http://www.ebi.ac.uk/interpro/entry/IPR000367 }}$ necessary for initiating parasite contact with epithelial surfaces and for the maintenance of cytoadhesion (reviewed in López et al., 2000). In addition to the above described mechanisms, proteins such as adhesins and CPs directly contribute to the parasite's cytoadherence (reviewed in López et al., 2000). It was theorized that the activity of CPs on cell surface molecules can elicit the synthesis of adhesins (reviewed in López et al., 2000). Nonetheless, one of the five known adhesin proteins, called AP65, was detected as overexpressed in the attenuated $H$. meleagridis strain by the SWATH experiment. It should be mentioned that AP65 is also a malic enzyme and the presence of an initial peptide sequence can direct this protein to the hydrogenosome where it exhibits a different function (Alderete et al., 1995; Engbring and Alderete, 1998). Taking into account that several other hydrogenosomal enzymes were detected as overexpressed in the attenuated parasite, the hydrogenosomal function of the above protein seems more likely.

\section{Survival Mechanisms}

Two NlpC/P60 superfamily domain-containing proteins showed increased expression in the virulent $H$. meleagridis parasite by the SWATH analysis. T. vaginalis possesses nine Clan CA, family C40 NlpC/P60 papain-like peptidases of the cysteine type that are involved in the cleavage of bacterial cell walls (Carlton et al., 2010; reviewed in Hirt et al., 2011). Considering that $H$. meleagridis utilizes co-cultivated E. coli DH5 $\alpha$ as food source, proteins like the above might contribute to its in vitro survival. In the same strain, the SWATH analysis identified an overexpressed lysin motif (LysM)-containing protein. This motif is found in enzymes that, in addition to chitin-binding, also degrade bacterial cell walls (InterPro entry ${ }^{6}$ ). Additionally, the LysM-containing protein, possesses a glycoside hydrolase 19 domain, typically found in chitinases (InterPro entry ${ }^{7}$ ). The cyst of the protozoan parasite Entamoeba spp. is encircled with chitin and its main element are proteins with chitin-binding domains, such as chitinases (Van Dellen et al., 2006). Under nutritional restricted conditions amoeboid $T$. vaginalis parasites, undergo a pseudocyst stage characterized by morphological changes such as round shape and internalized flagella (De Jesus et al., 2007). On the protein level, T. vaginalis pseudocyst stages were consistently overexpressing several actin proteins which were mapped to a single accession, PEPCK and a Rab11 isotype, whereas the expression of hydrogenosomal enzymes was shut down (De Jesus et al., 2007).

The overexpression of the above proteins in the gel images of the virulent $H$. meleagridis was accompanied by the absence of hydrogenosomal enzyme overexpression. In recent detailed investigations on $H$. meleagridis morphology, a genuine encystation process was not observed (Gruber et al., 2017). However, as it was the case for T. vaginalis, the flagellate was capable of defending itself by forming cystlike stages under adverse in vitro conditions characterized by rounded morphology and double cell membrane (Zaragatzki et al., 2010; Gruber et al., 2017). The upregulation

\footnotetext{
${ }^{6}$ https://www.ebi.ac.uk/interpro/entry/IPR018392

${ }^{7}$ https://www.ebi.ac.uk/interpro/entry/IPR000726
} 
of the above proteins in the virulent parasite, which is less adapted to the in vitro environment, indicates the existence of these defense mechanisms on a protein level.

\section{Glycolytic and Hydrogenosomal Proteins of the Carbohydrate Metabolism}

Overall, six enzymes of the glycolytic pathway (7 GAPDH spots, 5 PEPCK spots, 1 FBAL spot, 1 PPDK spot, 1 ADH spot, 1 PGlyM spot) and one protein (ADK protein) that acts in the hydrogenosome were shown to be overexpressed in the virulent strain by the gel-based and gel-free experiments. Except from the single GAPDH spot and $\mathrm{ADH}$, the detected overexpression of two other glycolytic proteins (2 PFK proteins, 1 enolase spot), as well as six hydrogenosomal enzymes ( 2 iron hydrogenase $64 \mathrm{kDa}$ proteins, 2 iron hydrogenase proteins, $1 \mathrm{NADH}$ dehydrogenase $51 \mathrm{kDa}$ protein, 1 malic enzyme) was the exclusive property of the attenuated parasite.

In amitochondriate anaerobic protozoa, such as $T$. vaginalis and $H$. meleagridis, carbohydrate metabolism is fermentative. The part of the glycolytic pathway that takes place in the cytoplasm results in pyruvate or malate production, which are further converted to hydrogen, carbon dioxide and acetate in double-membrane organelles called hydrogenosomes (De Jesus et al., 2007). Key hydrogenosomal enzymes that are involved in the latter process are the pyruvate ferredoxin oxidoreductase (PFOR), electron transporter ferredoxin (Fd), and hydrogenase (reviewed in Müller, 1993). Hydrogenosomal enzymes contain iron-sulfur clusters and iron is considered to be an essential element for their function, structure and transcription (De Jesus et al., 2007). Iron depletion can lead to the downregulation of several $T$. vaginalis hydrogenosomal enzymes, including Iron hydrogenase-1 (50 kDa), -2 (64 $\mathrm{kDa}$ ), and -3 (Beltrán et al., 2013). Interestingly, in this human parasite the downregulation of hydrogenosomal enzymes due to iron starvation was accompanied by an upregulation of certain glycolytic enzymes, including PEPCK (De Jesus et al., 2007), which was detected as overexpressed at multiple gel positions in the virulent $H$. meleagridis. The authors theorized that the overexpression of PEPCK under such conditions by $T$. vaginalis is part of an energy compensation mechanism (De Jesus et al., 2007), and one could argue that the same could apply for the virulent $H$. meleagridis. However, under in vitro conditions $H$. meleagridis should not experience a compromised hydrogenosomal activity due to iron deprivation since the fetal bovine serum, which is used as nutritional element, contains high levels of the ironstorage protein ferritin (Kakuta et al., 1997). Nonetheless, the in vitro environment might not be entirely optimal for this microaerophilic protozoan, considering that after each passage $H$. meleagridis comes in contact with atmospheric oxygen. It is known that $T$. vaginalis is sensitive to oxygen levels above those normally encountered in the host's vaginal environment (Ellis et al., 1994). In other trichomonads, such as Tritrichomonas foetus, the activities of PFOR and iron hydrogenase were negatively affected by oxygen with the latter showing higher sensitivity compared to the former (Lindmark and Müller, 1973). On the transcriptome level, oxygen protective molecules were found in both $H$. meleagridis strains, albeit the sequence encoding for superoxide dismutase (SOD), which is an important oxidative stress defense in $T$. vaginalis, was only reported in the attenuated one (Ellis et al., 1994; Mazumdar et al., 2017). Based on the above, the detected overexpression of hydrogenosomal enzymes in the attenuated strain might indicate the ability of this strain to cope better with the in vitro fluctuating oxygen levels. Alternatively, the fact that the overexpressed iron hydrogenases were mapped to different contigs can itself constitute a coping mechanism that protects hydrogenosomal metabolism from oxygen and might explain the higher adaptation of this strain to the in vitro environment.

\section{CONCLUSION}

This study corroborated, and to a greater extent supplemented the findings of the first $H$. meleagridis proteomic analysis that utilized conventional 2-DE (Monoyios et al., 2018). Results obtained from both 2D-DIGE experiments overlapped in the majority of identified proteins; however the SWATH MS analysis contributed to the identification of a completely new set of proteins. Overall, both gel-based and gel-free investigations displayed the poor adaptation of the low-passaged virulent strain to the in vitro conditions in comparison to the high-passaged attenuated strain. This was manifested by the upregulation of proteins related with stress response in the former and the overexpression of cell division proteins in the latter. Additionally, the upregulation of different components of the carbohydrate metabolism between these strains might further support this view. Molecules that are part of the virulence arsenal of other related parasites such as CPs and PFPs were detected in the virulent strain, a feature in large part identified by the SWATH MS experiment. Finally, both gel-based experiments identified multiple modifications of ubiquitous proteins such as actin and GAPDH in the virulent strain, which might indicate alternative functions and localizations. This finding was supported by the conventional 2-DE comparative study and should be addressed in greater detail by future investigations.

\section{DATA AVAILABILITY}

The mass spectrometry proteomic data were deposited to the ProteomeXchange Consortium via the PRIDE (Vizcaíno et al., 2016) partner-repository with the following accession numbers: PXD009332 for 2D-DIGE spot data obtained by MALDITOF/TOF, PXD009316 for 2D-DIGE spot data obtained by LCMS/MS and PXD009319 for the SWATH MS data and will be made publicly available.

Any other data supporting the conclusions of this manuscript can be made available by the authors, without undue reservation, to any qualified researcher upon request. 


\section{AUTHOR CONTRIBUTIONS}

IB, AM, and $\mathrm{MH}$ contributed conception and design of the study. MP assisted with 2D-Gels. $\mathrm{KN}, \mathrm{KH}$, and SS performed mass spectrometry analysis and wrote some material sections of the manuscript. AM, KN, and $\mathrm{KH}$ organized the database and performed the statistical analysis. AM and IB wrote the first draft of the manuscript. All authors contributed to manuscript revision, read, and approved the submitted version.

\section{FUNDING}

This project was funded by the Austrian Science Fund (FWF), grant no. P-25519-B25. The authors would like to thank the following researchers: Petra Ganas (Clinic for Poultry and Fish

\section{REFERENCES}

Alderete, J. F., O’Brien, J. L., Arroyo, R., Engbring, J. A., Musatovova, O., Lopez, O., et al. (1995). Cloning and molecular characterization of two genes encoding adhesion proteins involved in Trichomonas vaginalis cytoadherence. Mol. Microbiol. 17, 69-83. doi: 10.1111/j.1365-2958.1995.mmi_17010069.x

Amin, A., Nöbauer, K., Patzl, M., Berger, E., Hess, M., and Bilic, I. (2012). Cysteine peptidases, secreted by Trichomonas gallinae, are involved in the cytopathogenic effects on a permanent chicken liver cell culture. PLoS ONE 7:e37417. doi: 10.1371/journal.pone.0037417

Arnal, L., Grunert, T., Cattelan, N., de Gouw, D., Villalba, M. I., Serra, D. O., et al. (2015). Bordetella pertussis isolates from argentinean whooping cough patients display enhanced biofilm formation capacity compared to Tohama I reference strain. Front. Microbiol. 6:1352. doi: 10.3389/fmicb.2015.01352

Bachman, H., Nicosia, J., Dysart, M., and Barker, T. H. (2015). Utilizing fibronectin integrin-binding specificity to control cellular responses. Adv. Wound Care 4, 501-511. doi: 10.1089/wound.2014.0621

Baggerman, G., Vierstraete, E., De Loof, A., and Schoofs, L. (2005). Gel-based versus gel-free proteomics: a review. Comb. Chem. High Throughput Screen. 8, 669-677. doi: 10.2174/138620705774962490

Beltrán, N. C., Horváthová, L., Jedelský, P. L., Šedinová, M., Rada, P., Marcinčiková, M., et al. (2013). Iron-induced changes in the proteome of Trichomonas vaginalis hydrogenosomes. PLOS ONE 8:e65148. doi: 10.1371/journal.pone.0065148

Benjamini, Y., and Hochberg, Y. (1995). Controlling the false discovery rate - a practical and powerful approach to multiple testing. J. R. Stat. Soc. 57, 289-300.

Berth, M., Moser, F. M., Kolbe, M., and Bernhardt, J. (2007). The state of the art in the analysis of two-dimensional gel electrophoresis images. Appl. Microbiol. Biotechnol. 76, 1223-1243. doi: 10.1007/s00253-007-1128-0

Blum, H., Gross, H. J., and Beier, H. (1989). The expression of the TMV-specific $30-\mathrm{kDa}$ protein in tobacco protoplasts is strongly and selectively enhanced by actinomycin. Virology 169, 51-61. doi: 10.1016/0042-6822(89)90040-8

Carlton, J. M., Malik, S.-B., Sullivan, S. A., Sicheritz-Ponten, T., Tang, P., and Hirt, R. P. (2010). “The genome of Trichomonas vaginalis," in Anaerobic Parasitic Protozoa: Genomics and Molecular Biology, eds C. G. Clark, P. J. Johnson, and R. D. Adam (Norfolk: Caister Academic Press), 45-81.

Cepicka, I., Hampl, V., and Kulda, J. (2010). Critical taxonomic revision of parabasalids with description of one new genus and three new species. Protist 161, 400-433. doi: 10.1016/j.protis.2009.11.005

Crutchfield, C. A., Thomas, S. N., Sokoll, L. J., and Chan, D. W. (2016). Advances in mass spectrometry-based clinical biomarker discovery. Clin. Proteomics 13, 1-12. doi: 10.1186/s12014-015-9102-9

De Jesus, J. B., Cuervo, P., Britto, C., Sabóia-Vahia, L., Costa E Silva-Filho, F., Borges-Veloso, A., et al. (2009). Cysteine peptidase expression in Trichomonas vaginalis isolates displaying high- and low-virulence phenotypes. J. Proteome Res. 8, 1555-1564. doi: 10.1021/pr8009066
Medicine, University of Veterinary Medicine Vienna, Austria) and Tom Grunert (Functional Microbiology Division, Institute for Microbiology, University of Veterinary Medicine Vienna) for their advice on specific subjects. Tatjana Svoboda (Functional Microbiology Division, Institute for Microbiology, University of Veterinary Medicine Vienna) and Ingrid Miller (Institute of Medical Biochemistry, Department of Biomedical Sciences, University of Veterinary Medicine Vienna) for their help in scanning the gels.

\section{SUPPLEMENTARY MATERIAL}

The Supplementary Material for this article can be found online at: https://www.frontiersin.org/articles/10.3389/fcimb. 2018.00407/full\#supplementary-material

De Jesus, J. B., Cuervo, P., Junqueira, M., Britto, C., Costa e Silva-Filho, F., Soares, M. J., et al. (2007). A further proteomic study on the effect of iron in the human pathogen Trichomonas vaginalis. Proteomics 7, 1961-1972. doi: $10.1002 /$ pmic. 200600797

Dominguez, R., and Holmes, K. C. (2011). Actin structure and function. Annu. Rev. Biophys. 40, 169-186. doi: 10.1146/annurev-biophys-042910-155359

Ellis, J. E., Yarlett, N., Cole, D., Humphreys, M. J., and Lloyd, D. (1994). Antioxidant defences in the microaerophilic protozoan Trichomonas vaginalis: comparison of metronidazole-resistant and sensitive strains. Microbiology 140, 2489-2494. doi: 10.1099/13500872-140-9-2489

Engbring, J. A., and Alderete, J. F. (1998). Three genes encode distinct AP33 proteins involved in Trichomonas vaginalis cytoadherence. Mol. Microbiol. 28, 305-313. doi: 10.1046/j.1365-2958.1998.00784.x

Ganas, P., Liebhart, D., Glösmann, M., Hess, C., and Hess, M. (2012). Escherichia coli strongly supports the growth of Histomonas meleagridis, in a monoxenic culture, without influence on its pathogenicity. Int. J. Parasitol. 42, 893-901. doi: 10.1016/j.ijpara.2012.07.007

Gharahdaghi, F., Weinberg, C. R., Meagher, D. A., Imai, B. S., and Mische, S. M. (1999). Mass spectrometric identification of proteins from silverstained polyacrylamide gel: a method for the removal of silver ions to enhance sensitivity. Electrophoresis 20, 601-605. doi: 10.1002/(SICI)15222683(19990301)20:3<601::AID-ELPS601>3.0.CO;2-6

Gillet, L. C., Navarro, P., Tate, S., Röst, H., Selevsek, N., Reiter, L., et al. (2012). Targeted data extraction of the MS/MS spectra generated by data-independent acquisition: a new concept for consistent and accurate proteome analysis. Mol. Cell. Proteomics 11, 1-17. doi: 10.1074/mcp.O111.016717

González-Miguel, J., Morchón, R., Siles-Lucas, M., and Simón, F. (2015). Fibrinolysis and proliferative endarteritis: two related processes in chronic infections? The model of the blood-borne pathogen Dirofilaria immitis. PLoS ONE 10:e124445. doi: 10.1371/journal.pone.0124445

Görg, A., Drews, O., Lück, C., Weiland, F., and Weiss, W. (2009). 2-DE with IPGs. Electrophoresis 30, 122-132. doi: 10.1002/elps.200900051

Görg, A., Weiss, W., and Dunn, M. J. (2004). Current two-dimensional electrophoresis technology for proteomics. Proteomics 4, 3665-3685. doi: 10.1002/pmic.200401031

Gruber, J., Ganas, P., and Hess, M. (2017). Long-term in vitro cultivation of Histomonas meleagridis coincides with the dominance of a very distinct phenotype of the parasite exhibiting increased tenacity and improved cell yields. Parasitology 144, 1253-1263. doi: 10.1017/S0031182017 000646

Gruber, J., Pletzer, A., and Hess, M. (2018). Cholesterol supplementation improves growth rates of Histomonas meleagridis in vitro. Exp. Parasitol. 185, 53-61. doi: 10.1016/j.exppara.2018.01.007

Hawgood, S., Derrick, M., and Poulain, F. (1998). Structure and properties of surfactant protein B. Biochim. Biophys. Acta 1408, 150-160. doi: 10.1016/S0925-4439(98)00064-7 
Hess, M. (2017). Commensal or pathogen - a challenge to fulfil Koch's postulates. Br. Poult. Sci. 58, 1-12. doi: 10.1080/00071668.2016.1245849

Hess, M., Kolbe, T., Grabensteiner, E., and Prosl, H. (2006). Clonal cultures of Histomonas meleagridis, Tetratrichomonas gallinarum and a Blastocystis sp. established through micromanipulation. Parasitology 133, 547-554. doi: $10.1017 /$ S0031182006000758

Hess, M., Liebhart, D., Bilic, I., and Ganas, P. (2015). Histomonas meleagridis-new insights into an old pathogen. Vet. Parasitol. 208, 67-76. doi: 10.1016/j.vetpar.2014.12.018

Hess, M., and McDougald, L. R. (2013). "Histomoniasis (Blackhead) and other protozoan diseases of the intestinal tract," in Diseases of Poultry, ed D. E. Swayne (Ames, IA: Wiley-Blackwell; John Wiley \& Sons, Inc publication), 1172-1178.

Hirt, R. P., de Miguel, N., Nakjang, S., Dessi, D., Liu, Y. C., Diaz, N., et al. (2011). "Trichomonas vaginalis pathobiology: new insights from the genome sequence," in Advances in Parasitology, eds D. Rollinson and S. I. Hay (London: Elsevier Ltd.), 87-140.

Huang, K. Y., Chen, Y. Y. M., Fang, Y. K., Cheng, W. H., Cheng, C. C., Chen, Y. C., et al. (2014). Adaptive responses to glucose restriction enhance cell survival, antioxidant capability, and autophagy of the protozoan parasite Trichomonas vaginalis. Biochim. Biophys. Acta 1840, 53-64. doi: 10.1016/j.bbagen.2013.08.008

Huang, Q., Yang, L., Luo, J., Guo, L., Wang, Z., Yang, X., et al. (2015). SWATH enables precise label-free quantification on proteome scale. Proteomics 15, 1215-1223. doi: 10.1002/pmic.201400270

Hynes, R. O., and Yamada, K. M. (1982). Fibronectins: multifunctional modular glycoproteins. J. Cell Biol. 95, 369-377. doi: 10.1083/jcb.95.2.369

Jiménez, C. R., Huang, L., Qiu, Y., and Burlingame, A. L. (2001). "In-gel digestion of proteins for MALDI-MS fingerprint mapping," in Current Protocols in Protein Science, eds J. E. Coligan, B. M. Dunn, D. W. Speicher, and P. T, Wingfield (Brooklyn, NY: John Wiley \& Sons, Inc.), 16.4.1-16.4.5.

Kakuta, K., Orino, K., Yamamoto, S., and Watanabe, K. (1997). High levels of ferritin and its iron in fetal bovine serum. Comp. Biochem. Physiol. 118A, 165-169. doi: 10.1016/S0300-9629(96)00403-3

Kumar, G., Hummel, K., Ahrens, M., Menanteau-Ledouble, S., Welch, T. J., Eisenacher, M., et al. (2016). Shotgun proteomic analysis of Yersinia ruckeri strains under normal and iron-limited conditions. Vet. Res. 47, 1-13. doi: 10.1186/s13567-016-0384-3

Lama, A., Kucknoor, A., Mundodi, V., and Alderete, J. F. (2009). Glyceraldehyde3-phosphate dehydrogenase is a surface-associated, fibronectin-binding protein of Trichomonas vaginalis. Infect. Immun. 77, 2703-2711. doi: 10.1128/IAI.00157-09

Liebhart, D., Ganas, P., Sulejmanovic, T., and Hess, M. (2017). Histomonosis in poultry: previous and current strategies for prevention and therapy. Avian Pathol. 46, 1-18. doi: 10.1080/03079457.2016.1229458

Liebhart, D., Sulejmanovic, T., Grafl, B., Tichy, A., and Hess, M. (2013). Vaccination against histomonosis prevents a drop in egg production in layers following challenge. Avian Pathol. 42, 79-84. doi: 10.1080/03079457.2012.760841

Liebhart, D., Zahoor, M. A., Prokofieva, I., and Hess, M. (2011). Safety of avirulent histomonads to be used as a vaccine determined in turkeys and chickens. Poult. Sci. 90, 996-1003. doi: 10.3382/ps.2010-01255

Lindmark, D. G., and Müller, M. (1973). Hydrogenosome, a cytoplasmic organelle of the anaerobic flagellate Tritrichomonas foetus, and its role in pyruvate metabolism. J. Biol. Chem. 248, 7724-7728.

López, L. B., De Melo Braga, M. B., López, J. O., Arroyo, R., and Costa e Silva Filho, F. (2000). Strategies by which some pathogenic trichomonads integrate diverse signals in the decision-making process. An. Acad. Bras. Cienc. 72, 173-186. doi: $10.1590 /$ S0001-37652000000200006

Mazumdar, R., Endler, L., Monoyios, A., Hess, M., and Bilic, I. (2017). Establishment of a de novo reference transcriptome of Histomonas meleagridis reveals basic insights about biological functions and potential pathogenic mechanisms of the parasite. Protist 168, 663-685. doi: 10.1016/j.protis.2017.09.004

McDougald, L. R. (2005). Blackhead disease (Histomoniasis) in poultry : a critical review. Avian Dis. 49, 462-476. doi: 10.1637/7420-081005R.1

Miller, I. (2012). "Application of 2D DIGE in animal proteomics," in Difference Gel Electrophoresis (DIGE): Methods and Protocols. Series: Methods in Molecular
Biology, eds R. Cramer and R. Westermeier (New York, NY; Dordrecht; Heidelberg; London: Humana Press; Springer Science+ Business Media, LLC), 373-396.

Miller, I., and Gemeiner, M. (1992). Two-dimensional electrophoresis of cat sera: protein identification by cross reacting antibodies against human serum proteins. Electrophoresis 13, 450-453. doi: 10.1002/elps.1150130193

Mitra, B. N., Saito-Nakano, Y., Nakada-Tsukui, K., Sato, D., and Nozaki, T. (2007). Rab11B small GTPase regulates secretion of cysteine proteases in the enteric protozoan parasite Entamoeba histolytica. Cell. Microbiol. 9, 2112-2125. doi: 10.1111/j.1462-5822.2007.00941.x

Monoyios, A., Patzl, M., Schlosser, S., Hess, M., and Bilic, I. (2018). Unravelling the differences : comparative proteomic analysis of a clonal virulent and an attenuated Histomonas meleagridis strain. Int. J. Parasitol. 48, 145-157. doi: 10.1016/j.jpara.2017.08.017

Müller, M. (1993). The hydrogenosome. J. Gen. Microbiol. 139, 2879-2889. doi: 10.1099/00221287-139-12-2879

Oliveros, J. C. (2015). Venny. An Interactive Tool for Comparing Lists With Venn's Diagrams. Available online at: http://bioinfogp.cnb.csic.es/tools/venny/index. html

Petrak, J., Ivanek, R., Toman, O., Cmejla, R., Cmejlova, J., Vyoral, D., et al. (2008). Déjà vu in proteomics. A hit parade of repeatedly identified differentially expressed proteins. Proteomics 8, 1744-1749. doi: 10.1002/pmic.200700919

Pham, A. D., Mast, J., Magez, S., Goddeeris, B. M., and Carpentier, S. C. (2016). The enrichment of Histomonas meleagridis and its pathogen-specific protein analysis : a first step to shed light on its virulence. Avian Dis. 60, 628-636. doi: 10.1637/11389-021016-Reg.1

Plow, E. F., Herren, T., Redlitz, A., Miles, L. A., and Hoover-Plow, J. L. (1995). The cell biology of the plasminogen system. FASEB J. 9, 939-945. doi: 10.1096/fasebj.9.10.7615163

R Development Core Team (2014). R: A Language and Environment for Statistical Computing. R Foundation for Statistical Computing, Vienna, Austria. Available online at: http://www.R-project.org/

Rabilloud, T., Chevallet, M., Luche, S., and Lelong, C. (2010). Two-dimensional gel electrophoresis in proteomics: past, present and future. J. Proteomics 73, 2064-2077. doi: 10.1016/j.jprot.2010.05.016

Rosset, I., Tasca, T., Tessele, P. M., and De Carli, G. A. (2002). Scanning electron microscopy in the investigation of the in vitro hemolytic activity of Trichomonas vaginalis. Parasitol. Res. 88, 356-359. doi: 10.1007/s00436-001-0555-6

Shannon, P., Markiel, A., Ozier, O., Baliga, N. S., Wang, J. T., Ramage, D., et al. (2003). Cytoscape: a software environment for integrated models of biomolecular interaction networks. Genome Res. 13, 2498-2504. doi: 10.1101/gr.1239303

Shevchenko, A., Wilm, M., Vorm, O., and Mann, M. (1996). Mass spectrometric sequencing of proteins from silver-stained polyacrylamide gels. Anal. Chem. 68, 850-858. doi: $10.1021 /$ ac950914h

Singh, A., Weissenböck, H., and Hess, M. (2008). Histomonas meleagridis: immunohistochemical localization of parasitic cells in formalin-fixed, paraffinembedded tissue sections of experimentally infected turkeys demonstrates the wide spread of the parasite in its host. Exp. Parasitol. 118, 505-513. doi: 10.1016/j.exppara.2007.11.004

Sitek, B., Scheibe, B., Jung, K., Schramm, A., and Stühler, K. (2006). "Difference Gel Electrophoresis (DIGE): the next generation of two-dimensional gel electrophoresis for clinical research," in Methods and Principles in Medicinal Chemistry: Proteomics in Drug Research, eds M. Hamacher, K. Marcus, K. Stühler, A. van Hall, B. Warscheid, and H. Meyer (Weinheim: Wiley-VCH Verlag GmbH \& Co. KGaA, Weinheim), 33-55.

Sulejmanovic, T., Liebhart, D., and Hess, M. (2013). In vitro attenuated Histomonas meleagridis does not revert to virulence, following serial in vivo passages in turkeys or chickens. Vaccine 31, 5443-5450. doi: 10.1016/j.vaccine.2013.08.098

Taylor, G. K., and Goodlett, D. R. (2005). Rules governing protein identification by mass spectrometry. Rapid Commun. Mass Spectrom. 19, 3420-3420. doi: $10.1002 / \mathrm{rcm} .2225$

Tyzzer, E. E. (1919). Developmental phases of the protozoon of "Blackhead" in turkeys. J. Med. Res. 40, 1-30.3.

Ungermann, C., and Langosch, D. (2005). Functions of SNAREs in intracellular membrane fusion and lipid bilayer mixing. J. Cell Sci. 118, 3819-3828. doi: $10.1242 /$ jcs. 02561 
Van Dellen, K. L., Chatterjee, A., Ratner, D. M., Magnelli, P. E., Cipollo, J. F., Steffen, M., et al. (2006). Unique posttranslational modifications of chitinbinding lectins of Entamoeba invadens cyst walls. Eukaryot. Cell 5, 836-848. doi: 10.1128/EC.5.5.836-848.2006

Vizcaíno, J. A., Csordas, A., Del-Toro, N., Dianes, J. A., Griss, J., Lavidas, I., et al. (2016). 2016 update of the PRIDE database and its related tools. Nucleic Acids Res. 44, D447-D456. doi: 10.1093/nar/gkw880

Zaragatzki, E., Hess, M., Grabensteiner, E., Abdel-Ghaffar, F., Al-Rasheid, K. A. S., and Mehlhorn, H. (2010). Light and transmission electron microscopic studies on the encystation of Histomonas meleagridis. Parasitol. Res. 106, 977-983. doi: 10.1007/s00436-010-1777-2
Conflict of Interest Statement: The authors declare that the research was conducted in the absence of any commercial or financial relationships that could be construed as a potential conflict of interest.

Copyright (๔) 2018 Monoyios, Hummel, Nöbauer, Patzl, Schlosser, Hess and Bilic. This is an open-access article distributed under the terms of the Creative Commons Attribution License (CC BY). The use, distribution or reproduction in other forums is permitted, provided the original author(s) and the copyright owner(s) are credited and that the original publication in this journal is cited, in accordance with accepted academic practice. No use, distribution or reproduction is permitted which does not comply with these terms. 